Article

\title{
The 5D Fully-Covariant Theory of Gravitation and Its Astrophysical Applications
}

\section{Tianxi Zhang}

Department of Physics, Alabama A \& M University, 4900 Meridian Street, Normal, AL 35762, USA;

E-Mail: tianxi.zhang@aamu.edu; Tel.: +256-372-8106; Fax: +256-372-5622

Academic Editor: Antonaldo Diaferio

Received: 16 October 2014 / Accepted: 15 December 2014 / Published: 26 December 2014

\begin{abstract}
In this paper, we comprehensively review the five-dimensional (5D) fully-covariant theory of gravitation developed by Zhang two decades ago and its recent applications in astrophysics and cosmology. This 5D gravity describes not only the fields, but also the matter and its motion in a 5D spacetime. The greatest advantage of this theory is that there does not exist any unknown parameter, so that we can apply it to explain astrophysical and cosmological issues by quantitatively comparing the results obtained from it with observations and to predict new effects that could not be derived from any other gravitational theories. First, the 5D covariant description of matter and its motion enabled Zhang to analytically derive the fifteenth component of the 5D energy-momentum tensor of matter $\left(\bar{T}^{44}\right)$, which significantly distinguishes this 5D gravity from other 5D gravitational theories that usually assumed a $\bar{T}^{44}$ with an unknown parameter, called the scalar charge $s$, and, thus, to split the 5D covariant field equation into $(4+1)$ splitting form as the gravitational, electromagnetic, and scalar field equations. The gravitational field equation turns into the 4D Einstein's field equation of general relativity if the scalar field is equal to unity. Then, Zhang solved the field equations and obtained an exact static spherically-symmetric external solution of the gravitational, electromagnetic and scalar fields, in which all integral constants were completely determined with a perfect set of simple numbers and parameters that only depend on the mass and electric charge of the matter, by comparing with the obtained weak internal solution of the fields at a large radial distance. In the Einstein frame, the exact field solution obtained from the 5D fully-covariant theory of gravitation reduces to the Schwarzschild solution when the matter is electrically neutral and the fields are weak in strength. This guarantees that the four fundamental tests (light deflection, gravitational redshift, perihelion advance and radar echo delay) of the 4D Einstein's general relativity in the case of weak fields are also the tests of the 5D fully-covariant theory of gravitation. In
\end{abstract}


the case of strong fields, especially when the matter is highly charged, however, the results from the 5D fully-covariant theory of gravitation are significantly different from the 4D Einstein's general relativity. Applying this 5D gravity and its exact field solution, Zhang has recently developed a new redshift mechanism, called electric redshift, a new supernova explosion mechanism with gravitational field shielding, a new gravitationless black hole model, a modified neutron star mass-radius relation, a modified Friedmann equation for the accelerating universe, and so on. This paper provides an overview of this 5D fully-covariant theory of gravitation, including also its solution properties and astrophysical applications.

Keywords: Kaluza-Klein theory; classical black hole; neutron star; quasars

\section{Introduction}

To unify Einstein's general theory of relativity and Maxwell's theory of electromagnetism, in 1921, Kaluza proposed a five-dimensional (5D) unification theory [1]. A symmetric 5D spacetime metric has fifteen independent components. The first through tenth metric components describe the gravitational field; the eleventh through fourteenth metric components describe the electromagnetic field; and the fifteenth metric component is kept as a constant $l$. All field variables are explicitly independent of the fifth coordinate $x^{4}$ of the 5D spacetime. Then, Klein and other scientists further developed the 5D unification theory in the aspects of space structure, field covariance and tensor transformations [2-6]. With these efforts, people have understood that the 5D spacetime is composed of the normal 4D spacetime and a 1D small circular space with a radius of $\sim 10^{-33} \mathrm{~cm}$ [7]. Because the fifth dimension is circular, compact and small, the early 5D unification theory, which is usually called the Kaluza-Klein (KK) theory, does not conflict with our perspective of 4D spacetime. Mathematically, the early 5D KK theory successfully unified Einstein's general theory of relativity and Maxwell's theory of electromagnetism, but the unification did not produce any new effect in physics.

Taking into account Dirac's idea of a varying gravitational parameter [8], theorists had considered the fifteenth metric component to be a variable rather than a constant [9-11]. This variable corresponds to a new field, called the scalar field $\Phi$, which is governed by the fifteenth field equation (i.e., the scalar field equation). The 5D KK theory with a scalar field (5D KK $\Phi$ theory) produces new effects, such as the space or vacuum polarization $[12,13]$. The $5 \mathrm{D}$ KK $\Phi$ theory has been applied to study various physical issues, such as Planck mass [14], particle motion [15-17], charge-to-mass ratio [18-20], experimental tests [16,19], comparison with the Brans-Dicke theory [21], magnetic monopoles [22-25], cosmological phenomena [26-30], atomic properties [31] and other gravitational and electromagnetic phenomena [32]. The analyses for most of these physical issues must rely on a static, spherically-symmetric field solution of the $5 \mathrm{D} \mathrm{KK} \Phi$ theory.

Various static, spherically-symmetric field solutions of the $5 \mathrm{D} K K \Phi$ theory had been obtained [16,20,22,23,33-38]. However, all of these field solutions contained one or more undetermined constants. For instance, Vladimirov and Kislov [16] introduced a scalar field by multiplying $\Phi^{2}$ with 
the metric of the early KK theory. They obtained a static, spherically-symmetric field external solution with a unknown parameter, named the scalar charge $s$, which has no observational support. According to the field solution, they calculated the perihelion precessions of planets and the deflection of star light by the Sun. The results were shown to be consistent with Einstein's general theory of relativity and experimental measurements, if the scalar charge is chosen to be zero. In [33], Chodos and Detweiler developed a 5D KK $\Phi$ theory by assuming the 5D metric with a spacelike killing vector. They derived the gravitational, electromagnetic and scalar field equations with the projection method and exactly solved the set of field equations with two independent constants. To determine these constants, they also used the scalar charge to assume the fifteenth component of the $5 \mathrm{D}$ energy-momentum tensor $\bar{T}^{44}=s \rho$, where $\rho$ is the mass density. The unknown scalar charge leads to all predictions obtained from the $5 \mathrm{D} K \Phi$ theory being indecisive and the roles of the scalar field unsettled. Some other static solutions of the 5D KK $\Phi$ theory generalized from the Schwarzschild solution also have two or more constants undetermined [36-38]. Using a field solution with one or more undetermined constant or unknown parameter, we cannot obtain any conclusive result for a specific problem and, hence, cannot meaningfully compare the 5D KK $\Phi$ theory with other gravitational theories and experimental measurements [39,40].

To find a field solution for 5D gravity that does not include any undetermined constant, like the Schwazschild solution, the fifteenth component of the 5D energy-momentum tensor must be analytically derived, rather than assumed usually with a unknown parameter. Zhang [41] developed a 5D fully-covariant $\mathrm{KK} \Phi$ theory by describing not only the fields, but also the matter and its motion to be covariant in the 5D spacetime without assuming a scalar charge (see also [42,43]). The fifteenth component of the 5D energy-momentum tensor analytically derived was given by $\bar{T}^{44}=$ $\rho \alpha^{2} /\left(\Phi^{2} \sqrt{\Phi^{2}+\alpha^{2}}\right)$, where the charge-mass ratio $\alpha$ was defined by Equation (59). For this 5D fully-covariant theory of gravitation (or say, 5D fully-covariant $\mathrm{KK} \Phi$ theory), Zhang further obtained an exact static spherically-symmetric field external solution without any undetermined constant or unknown parameter $[41,44,45]$. All integral constants in the field solution of this 5D fully-covariant theory of gravitation were nicely determined by a perfect set of simple numbers and parameters that only depend on the mass and electric charge of matter. In the Einstein frame, the field solution reduces to the Schwarzschild solution when the fields are weak and the matter is electrically neutral. This guarantees that the four fundamental tests of Einstein's general theory of relativity in the case of weak fields, where the gravitational energy of a test particle is much less than its rest energy, are also the tests of the 5D fully-covariant $\mathrm{KK} \Phi$ theory. The four fundamental tests usually refer to the gravitational redshift of light from the Sun, the deflection of star light by the Sun, the perihelion precession of planets and the time delay of radar echoes. However, in the case of strong fields, especially when the matter is not only massive and compact, but also significantly charged electrically, the 5D fully-covariant $\mathrm{KK} \Phi$ theory can, in accordance with the field solution, predict results that are significantly different from Einstein's general theory of relativity with the Schwarzschild solution. In this case, the 5D fully-covariant KK $\Phi$ theory is competitive and has a wide application in strong field astrophysics.

Recently, Zhang has applied the 5D fully-covariant $\mathrm{KK} \Phi$ theory to describe and explain the strong field astrophysical issues. First, he has developed a new redshift mechanism, called electric redshift [45]. The results indicated that an electrically-charged, massive, compact object can significantly shift the light that is emitted from the object toward the red end in comparison with the gravitational redshift. For 
an electrically-charged, compact object with density and mass comparable to that of a neutron star, the electric redshift can be as great as that of quasars. Then, he has developed a new supernova explosion mechanism with gravitational field shielding [46] and a new gravitationless black hole model [47]. It is shown that a dense compact neutral core of a star, when it collapses to a critical density, suddenly turns off or shields its gravitational field. The core, if its mass exceeds an upper limit, directly collapses into a black hole. Otherwise, the extremely large pressure, as the gravity is turned off, immediately stops the collapse and drives the mantle material of the supernova to move outward, which leads to an impulsive explosion and forms a neutron star as a remnant. A neutron star can further evolve into a black hole when it accretes enough matter from a companion star, such that the total mass exceeds a lower limit. The black hole in the 5D fully-covariant $\mathrm{KK} \Phi$ theory is gravitationless at the surface, because the scalar field is infinitely strong, which varies the equivalent gravitational constant to zero. In general, a star, at the end of its evolution, is relatively harder to collapse into a gravitationless KK black hole than a strong gravitational Schwarzschild black hole. This is consistent with the recent observation of some very massive stars forming neutron stars, rather than the expected black holes [48-50]. In addition, a gravitationless KK black hole should be able to more easily generate jets than a Schwarzschild black hole.

In this paper, we will fully describe the 5D fully-covariant $\mathrm{KK} \Phi$ theory and its astrophysical application and comprehensively analyze the characteristics and new physical effects obtained from this unification theory. In Section 2, we will detail the development of the 5D fully-covariant KK $\Phi$ theory. Both the fields and matter, including its motion, will be described as 5D covariant. In Section 3, we will examine the 5D energy-momentum tensor and field equation to derive all fifteen components of the 5D energy-momentum tensor and to decouple the 5D field equation to have the gravitational, electromagnetic and scalar field equations without introducing any unknown parameter. In Section 4, we will derive the equation of motion according to the 5D fully-covariant $\mathrm{KK} \Phi$ theory. In Section 5, we will solve the field equation and obtain an exact static spherically-symmetric field external solution of the present 5D KK $\Phi$ theory and then determine all integration constants according to the internal field weak limits. In Section 6, we will analyze the properties of the exact static spherically-symmetric field external solution, such as the singularity, vacuum polarization, frame transformation and charge and scalar field effects on light, gravity and the electric field, including the electric redshift mechanism, the gravitational field shielding concept and the gravitationless black hole model. In the last section, we will give our discussions and conclusions.

\section{The 5D Fully-Covariant KK $\Phi$ Theory}

\subsection{The 5D Spacetime Metric and Fields}

To describe the geometric structure of a spacetime, one usually defines an orthogonal frame (i.e., vielbein) attached to each point in the spacetime. In the present 5D fully-covariant KK $\Phi$ theory [41-43], the $5 \mathrm{D}$ spacetime vielbein is represented by the following $(4+1)$ splitting form,

$$
\bar{e}_{\alpha}^{A}=\left(\begin{array}{cc}
e_{\mu}^{i} & 0 \\
q l \Phi A_{\mu} & l \Phi
\end{array}\right),
$$


and its inverse is given by:

$$
\bar{e}_{A}^{\alpha}=\left(\begin{array}{cc}
e_{i}^{\mu} & 0 \\
-q A_{i} & l^{-1} \Phi^{-1}
\end{array}\right),
$$

where $e_{\mu}^{i}$ and $e_{i}^{\mu}$ are the 4D spacetime vielbein and its inverse, respectively; $A_{\mu}$ is the 4D electromagnetic potential; $\Phi$ is the scalar field; $l$ is a constant that is usually chosen to be unity or absorbed into $\Phi ; q$ is a scale constant defined by $q l=2 \sqrt{G}$ with $G$ the gravitational constant. The 5D spacetime vielbein indices are integers represented by the uppercase Latin letters, $A, B$, etc., running through zero to four; while the 4D spacetime vierbein indices are integers represented by the lowercase Latin letters, $i, j$, etc., running through zero to three. The 5D spacetime coordinate indices are integers represented by the Greek letters, $\alpha, \beta$, etc., running through zero to four; while the 4D spacetime coordinate indices are integers represented by $\mu, \nu$, etc., running through zero to three. All 5D quantities are headed with a bar to distinguish them from the $4 \mathrm{D}$ quantities. If $\bar{e}_{\alpha}^{A}$ and $\bar{e}_{A}^{\alpha}$ are independent of the fifth coordinate $x^{4}$, then from the 5D KK field equation, one can derive Einstein's gravitational field equation of general relativity and Maxwell's electromagnetic field equation of classical electrodynamics. When $\Phi=1$, it reduces to the 5D spacetime vielbein of the early KK theory without a scalar field.

The 5D spacetime Lorentz metric is given by:

$$
\bar{\eta}_{A B}=\bar{\eta}^{A B}=\left(\begin{array}{ccccc}
-1 & 0 & 0 & 0 & 0 \\
0 & 1 & 0 & 0 & 0 \\
0 & 0 & 1 & 0 & 0 \\
0 & 0 & 0 & 1 & 0 \\
0 & 0 & 0 & 0 & 1
\end{array}\right) .
$$

From Equations (1)-(3), the 5D spacetime metric $\bar{g}_{\alpha \beta}$ and its inverse $\bar{g}^{\alpha \beta}$ can be split into the following $(4+1)$ forms, respectively:

$$
\begin{gathered}
\bar{g}_{\alpha \beta} \equiv \bar{\eta}_{A B} \bar{e}_{\alpha}^{A} \bar{e}_{\beta}^{B}=\left(\begin{array}{cc}
g_{\mu \nu}+q^{2} l^{2} \Phi^{2} A_{\mu} A_{\nu} & q l^{2} \Phi^{2} A_{\nu} \\
q l^{2} \Phi^{2} A_{\mu} & l^{2} \Phi^{2}
\end{array}\right), \\
\bar{g}^{\alpha \beta} \equiv \bar{\eta}^{A B} \bar{e}_{A}^{\alpha} \bar{E}_{B}^{\beta}=\left(\begin{array}{cc}
g^{\mu \nu} & -q A^{\nu} \\
-q A^{\mu} & q^{2} A^{\lambda} A_{\lambda}+l^{-2} \Phi^{-2}
\end{array}\right) .
\end{gathered}
$$

Note that, when $l=1$ or absorbed into $\Phi$, the 5D spacetime metric Equation (4) is exactly the same as the 5D spacetime metric given by Overduin and Wesson [51]. When $\Phi=1$, we have the 5D spacetime metric of the early KK theory that does not have a scalar field.

The 5D Einstein curvature tensor depends on the 5D spacetime metric with vielbein indices through the following set of formulae in accordance with the Riemann geometrical theory,

$$
\bar{G}^{A B}=\bar{R}^{A B}-\frac{1}{2} \bar{\eta}^{A B} \bar{R}
$$

where the 5D spacetime Ricci tensor $\bar{R}_{B C}$, its inverse $\bar{R}^{B C}$ and its curvature scalar $\bar{R}$ are given by:

$$
\begin{gathered}
\bar{R}_{B C}=\bar{\Gamma}_{B C, A}^{A}-\bar{\Gamma}_{B A, C}^{A}+\bar{\Gamma}_{D A}^{A} \bar{\Gamma}_{B C}^{D}-\bar{\Gamma}_{D C}^{A} \bar{\Gamma}_{B A}^{D}+\bar{\Gamma}_{B D}^{A} \bar{\Gamma}_{A C}^{D} \\
\bar{R}^{B C}=\bar{\eta}^{F B} \bar{\eta}^{E C} \bar{R}_{F E},
\end{gathered}
$$




$$
\bar{R}=\bar{\eta}_{A B} \bar{R}^{A B}
$$

Here, the 5D Christoffel symbol $\bar{\Gamma}_{B C}^{A}$ is defined by:

$$
\bar{\Gamma}_{B C}^{A}=\frac{1}{2}\left(\bar{B}_{B C}^{A}+\bar{B}_{B C}{ }^{A}-\bar{B}_{C B}^{A}\right),
$$

with:

$$
\bar{B}_{B C}^{A}=\left(\bar{e}_{\alpha, \beta}^{A}-\bar{e}_{\beta, \alpha}^{A}\right) \bar{e}_{B}^{\beta} \bar{e}_{C}^{\alpha},
$$

and:

$$
\begin{gathered}
\bar{B}_{B C}^{A}=\bar{B}_{B C}^{A}, \\
\bar{B}_{B C}{ }^{A}=\bar{\eta}^{A E} \bar{\eta}_{B F} \bar{B}_{C E}^{F}, \\
\bar{B}_{C B}^{A}=\bar{\eta}^{A E} \bar{\eta}_{C F} \bar{B}_{E B}^{F} .
\end{gathered}
$$

Substituting the 5D spacetime vielbein (1) and its inverse (2) into the above formula and using the 5D spacetime Lorentz metric (3), we obtain the 5D Einstein curvature tensor in the $(4+1)$ splitting form with the $4 \mathrm{D}$ vielbein indices as:

$$
\begin{gathered}
\bar{G}^{i j}=G^{i j}-2 G \Phi^{2}\left(F^{i k} F_{k}^{j}-\frac{1}{4} \eta^{i j} F^{k l} F_{k l}\right)-\frac{1}{\Phi}\left(\Phi^{, i ; j}-\eta^{i j} \Phi_{; k}^{, k}\right), \\
\bar{G}^{4 j}=\bar{G}^{j 4}=\sqrt{G} \Phi\left(F_{; k}^{j k}+\frac{3}{\Phi} F^{j k} \Phi_{, k}\right), \\
\bar{G}^{44}=\frac{3}{2} G \Phi^{2} F^{j k} F_{j k}-\frac{1}{2} R
\end{gathered}
$$

and other components are zero. Here, $F^{i j}=A^{j, i}-A^{i, j}$ is the electromagnetic field tensor, in which the symbol "," refers to the usual derivative; the symbol ";" refers to the covariant derivative; and $R$ is the 4D Ricci curvature scalar, which is related to the 5D Ricci curvature scalar $\bar{R}$ by,

$$
\bar{R}=R-G \Phi^{2} F^{j k} F_{j k}-\frac{2}{\Phi} \Phi_{; j}^{, j} .
$$

The 5D action of fields is usually represented as:

$$
\bar{S}(G)=\frac{1}{16 \pi \bar{G}} \int \sqrt{-\bar{g}} \bar{R} d x^{0} d x^{1} d x^{2} d x^{3} d x^{4}
$$

where $\bar{G}$ is a 5D coupling constant, $\bar{g}$ is the determinant of the 5D metric $\bar{g}_{\alpha \beta}$ and the fifth coordinate $x^{4}$ is integrated in the range of $0-1$. This section describes in the standard way the field and metric of the present $5 \mathrm{D} \mathrm{KK} \Phi$ theory.

\subsection{The 5D Covariant Description of Matter}

In the various types of $5 \mathrm{D} \mathrm{KK} \Phi$ theories developed so far, scientists have described the geometric tensors and the action of fields to be 5D covariant similar to what is shown in the above section. However, the 5D covariance of the energy-momentum tensor and action of matter has not yet been fully considered. All previous 5D theories did not define a 5D covariant density of matter and a 5D covariant velocity of motion, so they did not define a 5D covariant action of matter, in the 5D spacetime. The field 
equations were formulated in a 5D covariant form only through assuming a $5 \mathrm{D}$ covariant energy-momentum tensor. To assume the fifteenth component of the $5 \mathrm{D}$ energy-momentum tensor, one had to introduce an unknown parameter, the scalar charge $s$.

To develop a 5D fully-covariant $\mathrm{KK} \Phi$ theory, we must describe the matter and its motion in the 5D spacetime. We must define a 5D covariant action of matter from which one can derive a 5D covariant energy-momentum tensor, rather than having to assume it with an unknown parameter. For this purpose, in the 5D fully-covariant $\mathrm{KK} \Phi$ theory developed by Zhang [41] (see also [42,43]), the matter and its motion are described in the 5D spacetime by a 5D covariant density $\bar{\rho}$ and a 5D covariant velocity $\bar{u}^{\alpha}$, where $\bar{u}^{\alpha}$ is defined by:

$$
\bar{u}^{\alpha}=\frac{d x^{\alpha}}{\overline{d s}}
$$

and the line element of the 5D spacetime $\overline{d s}$ is given, in the coordinate indices, by:

$$
\overline{d s}^{2}=-\bar{g}_{\alpha \beta} d x^{\alpha} d x^{\beta}
$$

and, in the vielbein indices, by:

$$
\overline{d s}^{2}=-\eta_{A B} d q^{A} d q^{B}
$$

with $d q^{A}=\bar{e}_{\alpha}^{A} d x^{\alpha}$. The 5D current of matter can be written as:

$$
\bar{J}^{\alpha}=\bar{\rho} \bar{u}^{\alpha} .
$$

Given the 5D current of matter as a 5D conserved quantity, we have:

$$
\bar{J}_{; \alpha}^{\alpha}=0
$$

Then, in the 5D spacetime, the conserved quantity (energy of matter) of Equation (24) in a closed system can be represented as:

$$
\bar{M}=\int \bar{J}^{0} \sqrt{-\bar{g}} d x^{1} d x^{2} d x^{3} d x^{4}
$$

Here, $\bar{g}$ is the determinant of the metric tensor, $\bar{g}=\operatorname{det}\left(\bar{g}_{\alpha \beta}\right)$. Replacing $\sqrt{-\bar{g}}$ by $l \Phi \sqrt{-g_{00}} \sqrt{\gamma}$ with $\gamma$ the determinant of the 5D spacetime metric in the 3D space and integrating Equation (25) with respect to the fifth coordinate $x^{4}$ from zero to one, we have:

$$
\bar{M}=\int l \Phi \bar{\rho} f^{-1} \frac{d \tau}{d s} d V .
$$

where $f$ is defined by:

$$
f=\frac{\overline{d s}}{d s}
$$

and the time and volume elements of the 4D spacetime $d \tau$ and $d V$ are given, respectively, by:

$$
\begin{gathered}
d \tau=\int \sqrt{-g_{00}} d x^{0}, \\
d V=\int \sqrt{\gamma} d x^{1} d x^{2} d x^{3} .
\end{gathered}
$$


Using the line elements in both the 4D and 5D spacetimes, we can determine the factor $f$, which has never been studied in any other $\mathrm{KK} \Phi$ theory, as:

$$
f=\frac{1}{\sqrt{1+\left(\bar{w}^{4}\right)^{2}}}
$$

where $\bar{w}^{4}$ is the fifth component of the $5 \mathrm{D}$ velocity in the vielbein indices $\bar{w}^{A}$, which is given by:

$$
\bar{w}^{A} \equiv \frac{d q^{A}}{\bar{d} s}=\bar{e}_{\alpha}^{A} \frac{d x^{\alpha}}{\bar{d} s}=\bar{e}_{\alpha}^{A} \bar{u}^{\alpha} .
$$

In the general relativity of the 4D spacetime, matter is described by density $\rho$ and velocity $v^{\mu}$. The conserved energy of matter in the 4D spacetime is given by:

$$
M=\int \rho \frac{d \tau}{d s} d V
$$

where $d s$ is the line element of the 4D spacetime. Since both $\bar{M}$ and $M$ denote the conserved energy of matter and have the same dimension, we have $\bar{M}=M$. Then, from Equations (26) and (32), we obtain the relation between densities $\bar{\rho}$ and $\rho$,

$$
\bar{\rho}=l^{-1} \Phi^{-1} f \rho .
$$

This density relation can be used to split the 5D energy-momentum tensor into a $(4+1)$ expression. For the pressureless matter described by $\bar{\rho}$ and $\bar{u}_{\alpha}$, we can define the 5D action of matter in a way similar to general relativity as:

$$
\bar{S}(m)=-\int \bar{\rho} \sqrt{-\bar{g}} d x^{0} d x^{1} d x^{2} d x^{3} d x^{4}
$$

If we introduce a quantity of the form,

$$
\bar{P}^{\alpha}=\sqrt{-\bar{g}} \bar{J}^{\alpha}
$$

then we can rewrite Equation (24) as:

$$
\partial_{\alpha} \bar{P}^{\alpha}=0
$$

where $\partial_{\alpha} \equiv \partial / \partial x^{\alpha}$. That is to say, the quantity $\bar{P}^{\alpha}$ is independent of the 5D spacetime metric $\bar{g}^{\alpha \beta}$. Using $\bar{P}^{\alpha}$, we obtain the 5D covariant action of the pressureless matter as the following form:

$$
\bar{S}(m)=-\int \sqrt{-\bar{g}_{\alpha \beta} \bar{P}^{\alpha} \bar{P}^{\beta}} d x^{0} d x^{1} d x^{2} d x^{3} d x^{4} .
$$

All of above definitions and analyses were done in the same way as previously done in general relativity. For the conserved energy of matter derived from the present $5 \mathrm{D}$ KK $\Phi$ theory to be equal to that derived from general relativity, we have related the $5 \mathrm{D}$ covariant density of matter to the density observed in the 4D spacetime (Equation (33)). This also guarantees the gravitational field equation (Equation (64)) derived from the present 5D theory to be Einsteinian in the case of neutral matter and a constant scalar field.

It should be also noted that the matter description does not affect the field external solution. To obtain a weak field interior solution for low-density matter, a 5D pressureless matter approximation is sufficient. In general relativity, physicists have developed both the 4D pressureless matter model and 
the fluid model with a non-zero pressure. In the previous 5D theories, however, theorists had not yet developed a 5D covariant matter model. Matter and its motion were still described in the 4D spacetime. The only difference is that a scalar charge is introduced to assume the fifteenth energy-momentum tensor. In the present $5 \mathrm{D}$ theory, we have fully described matter and its motion in the 5D spacetime. We have successfully developed a 5D pressureless matter model and obtained significant results from this 5D fully-covariant $\mathrm{KK} \Phi$ theory. This section has uniquely described the matter, including its motion and the action of matter with 5D covariance.

\section{The 5D Energy-Momentum Tensors and Field Equations}

The total action of field and matter with 5D covariance is:

$$
\bar{S}(\text { total })=\overline{\mathrm{S}}(\mathrm{G})+\overline{\mathrm{S}}(\mathrm{m})
$$

Making use of the variation principle $\delta \bar{S}($ total $)=0$, we obtain the $5 \mathrm{D}$ field equation of the $5 \mathrm{D}$ fully-covariant $\mathrm{KK} \Phi$ theory as,

$$
\bar{G}_{\alpha \beta}=8 \pi G \bar{T}_{\alpha \beta}
$$

or in superscripts as:

$$
\bar{G}^{\alpha \beta}=8 \pi G \bar{T}^{\alpha \beta} .
$$

In the 5D vielbein indices form, we can rewrite Equation (40) as:

$$
\bar{G}^{A B}=8 \pi G \bar{T}^{A B}
$$

Here, we have chosen $\bar{G}=l G$ and defined the 5D energy-momentum tensor of matter by:

$$
\bar{T}_{\alpha \beta}=l \frac{2}{\sqrt{-\bar{g}}} \frac{\partial}{\partial \bar{g}^{\alpha \beta}}\left(\sqrt{-\bar{g}_{\alpha_{1} \beta_{1}} \bar{P}^{\alpha_{1}} \bar{P}^{\beta_{1}}}\right) .
$$

Using the following formulae:

$$
\frac{\partial \bar{g}_{\alpha_{1} \beta_{1}}}{\partial \bar{g}^{\alpha \beta}}=-\bar{g}_{\alpha \alpha_{1}} \bar{g}_{\beta \beta_{1}},
$$

and Equations (23), (33), (35) and (36), we obtain the 5D energy-momentum tensor as:

$$
\bar{T}_{\alpha \beta}=l \bar{\rho} \bar{u}_{\alpha} \bar{u}_{\beta}=\Phi^{-1} f^{-1} \rho \bar{v}_{\alpha} \bar{v}_{\beta},
$$

or:

$$
\bar{T}^{\alpha \beta}=l \bar{\rho} \bar{u}^{\alpha} \bar{u}^{\beta}=\Phi^{-1} f^{-1} \rho \bar{v}^{\alpha} \bar{v}^{\beta},
$$

where $\bar{v}^{\alpha}$ is defined by:

$$
\bar{v}^{\alpha} \equiv \frac{d x^{\alpha}}{d s}=f \bar{u}^{\alpha}
$$

Expressing $\bar{T}^{\alpha \beta}$ with the vielbein indices, we have:

$$
\bar{T}^{A B}=l \bar{\rho} \bar{w}^{A} \bar{w}^{B}=\Phi^{-1} f \rho \bar{w}^{A} \bar{w}^{B},
$$

which can be easily split into $(4+1)$ form as follows:

$$
\bar{T}^{i j}=\Phi^{-1} f^{-1} T^{i j},
$$




$$
\begin{gathered}
\bar{T}^{i 4}=\bar{T}^{4 i}=\Phi^{-1} \rho v^{i} \bar{w}^{4}, \\
\bar{T}^{44}=\Phi^{-1} f \rho \bar{w}^{4} \bar{w}^{4} .
\end{gathered}
$$

Here, we have used the following relations:

$$
\bar{w}^{i}=\bar{e}_{\alpha}^{i} \bar{u}^{\alpha}=\bar{e}_{\mu}^{i} \bar{u}^{\mu}=\bar{e}_{\mu}^{i} v^{\mu} f^{-1}=e_{\mu}^{i} v^{\mu} f^{-1}=v^{i} f^{-1} .
$$

The 4D energy-momentum tensor of matter $T^{i j}$ is given by:

$$
T^{i j}=\rho v^{i} v^{j}
$$

Then, the field equations can be divided into:

$$
\begin{gathered}
G^{i j}=8 \pi G \Phi^{-1} f^{-1} T^{i j}+2 G \Phi^{2}\left(F_{k}^{i k} F^{j}-\frac{1}{4} \eta^{i j} F^{k l} F_{k l}\right)+\frac{1}{\Phi}\left(\Phi^{, i ; j}-\eta^{i j} \Phi_{; k}^{, k}\right), \\
\left(F^{i k} \Phi^{3}\right)_{; k}=8 \pi \sqrt{G} \Phi \rho v^{i} \bar{w}^{4}, \\
\frac{3}{2} G \Phi^{2} F^{i j} F_{i j}-\frac{1}{2} R=8 \pi G \Phi^{-1} f \rho \bar{w}^{4} \bar{w}^{4} .
\end{gathered}
$$

Equation (54) should have the form of Maxwell's equation of electromagnetic fields. For this reason, we have:

$$
8 \pi \sqrt{G} \Phi \rho v^{i} \bar{w}^{4}=4 \pi j_{e}^{i}
$$

where $j_{e}^{i}$ is the $4 \mathrm{D}$ electric current density. For a uniform charged system with mass $m$ and electric charge $Q$, the electric current density $j_{e}^{i}$ can be written as:

$$
j_{e}^{i}=\frac{Q}{m} \rho v^{i}
$$

Substituting Equation (57) into Equation (56), we obtain the fifth component of the 5D covariant velocity of motion:

$$
\bar{w}^{4}=\frac{Q}{2 \sqrt{G} m} \Phi^{-1}=\alpha \Phi^{-1}
$$

where:

$$
\alpha=\frac{Q}{2 \sqrt{G} m} .
$$

Then, the factor $f$ (Equation (30)) becomes:

$$
f=\frac{\Phi}{\sqrt{\alpha^{2}+\Phi^{2}}}
$$

the fifteenth component of the 5D energy-momentum tensor $\bar{T}^{44}$ (Equation (50)) can be derived as:

$$
\bar{T}^{44}=\frac{\rho \alpha^{2}}{\Phi^{2} \sqrt{\alpha^{2}+\Phi^{2}}},
$$

and the field equations cam be simplified as:

$$
G^{i j}=8 \pi G \Phi^{-1} f^{-1} T^{i j}(m)+2 G \Phi^{2}\left(F^{i k} F_{k}^{j}-\frac{1}{4} \eta^{i j} F^{k l} F_{k l}\right)+\frac{1}{\Phi}\left(\Phi^{, i ; j}-\eta_{; j}^{i j} \Phi_{; k}^{, k}\right)
$$




$$
\begin{gathered}
\left(\Phi^{3} F^{i k}\right)_{; k}=4 \pi j_{e}^{i}, \\
\Phi_{; k}^{, k}=G \Phi^{3} F^{i j} F_{i j}+\frac{8 \pi G}{3} f^{-1}\left[T^{i}{ }_{i}(m)-\frac{2 \alpha^{2} \rho}{\Phi^{2}+\alpha^{2}}\right] .
\end{gathered}
$$

In the coordinate indices form, the field equations of the 5D fully-covariant $K K \Phi$ theory are:

$$
\begin{gathered}
G_{\nu}^{\mu}=8 \pi G \Phi^{-1}\left[f^{-1} T_{\nu}^{\mu}(m)+T_{\nu}^{\mu}(F)+T_{\nu}^{\mu}(\Phi)\right], \\
H_{; \nu}^{\mu \nu}=4 \pi j_{e}^{\mu}, \\
\Phi_{; \lambda}^{, \lambda}=G \Phi^{-3} H^{\mu \nu} H_{\mu \nu}+\frac{8 \pi G}{3} f^{-1}\left[T_{\mu}^{\mu}(m)-\frac{2 \alpha^{2} \rho}{\Phi^{2}+\alpha^{2}}\right],
\end{gathered}
$$

where:

$$
\begin{gathered}
T_{\nu}^{\mu}(m)=\rho v^{\mu} v_{\nu} \\
T_{\nu}^{\mu}(F)=\frac{\Phi^{3}}{4 \pi}\left(F^{\mu \lambda} F_{\nu \lambda}-\frac{1}{4} \delta_{\nu}^{\mu} F^{\lambda \rho} F_{\lambda \rho}\right), \\
T_{\nu}^{\mu}(\Phi)=\frac{1}{8 \pi G}\left(\Phi_{; \nu}^{, \mu}-\delta_{\nu}^{\mu} \Phi_{; \lambda}^{, \lambda}\right), \\
H^{\mu \nu}=\Phi^{3} F^{\mu \nu} .
\end{gathered}
$$

It is seen that, from this set of field equations, there is not any unknown parameter like the scalar charge in [33]. The parameter $f$ is a new factor, which is determined by the constant $\alpha$ and the scalar field $\Phi$. When the matter is electrically neutral $(\alpha=0)$, the factor $f$ becomes unity. The scalar field equation of the 5D fully-covariant $\mathrm{KK} \Phi$ theory is comparable to the governing equation of the Brans-Dicke theory with the dimensionless Brans-Dicke coupling constant $\omega=0$ for an asymptotic limitation [52]. When $\alpha=0$ and $\Phi=1$, Equation (65) becomes the 4D Einstein field equation. A scalar field (if $\Phi>1$ ) can significantly weaken both gravitational and electromagnetic fields of matter. In other words, it can effectively shield the gravity and polarize the space.

For the electromagnetic field equation (Equation (54)) derived from the present 5D theory to be Maxwellian, we determined the fifth component of the 5D covariant velocity of motion (Equation (58)) in terms of the charge-mass ratio and scalar field. We further determined the fifteenth component of the 5D energy-momentum tensor in terms of the fifth component of the 5D covariant velocity of motion (Equation (50)). Distinguishing from the previous 5D theories, which did not describe matter and its motion in the $5 \mathrm{D}$ spacetime, we call the present one a $5 \mathrm{D}$ fully-covariant $\mathrm{KK} \Phi$ theory or $5 \mathrm{D}$ fully-covariant gravity.

The scalar field in the 5D fully-covariant $\mathrm{KK} \Phi$ theory is governed by the scalar field equation (Equation (64) or Equation (67)) without any free parameter and, thus, completely determined from the solution rather than chosen arbitrarily. It deviates from (or reduces to) the $4 \mathrm{D}$ gravity, but is negligible in the case of weak fields and neutral matter, so that the 5D fully-covariant $\mathrm{KK} \Phi$ theory does not conflict with Newton's law of gravity and observations. The present 5D theory is permitted with the experimental point of view. It also predicts new effects when the fields, including the scalar field, are strong and, thus, has important applications in astrophysics with strong fields. The scalar field of all previous 5D theories needs to be stabilized for it to be permitted with the experimental point of view, but the present 5D theory does not need such constrains. We couple the matter with the scalar field through a 5D fully-covariant 
description of matter and its motion, rather than by a specific set of stabilization conditions. We develop this $5 \mathrm{D}$ theory from the $5 \mathrm{D}$ vielbein or the metric for the fields and the $5 \mathrm{D}$ covariant description of density and velocity for the matter and its motion.

\section{Equation of Motion}

Using the 5D Bianchi identity, $\bar{G}_{; B}^{A B}=0$, we can obtain the 5D equation of motion from the 5D Einstein field equation (Equation (41)) as:

$$
\bar{T}_{; B}^{A B}=0 .
$$

When $A=4$, we have that $d \alpha / d s=0$, and hence, $d \alpha / \bar{d} s=0$. This means that $\alpha$ is a constant of motion in the $4 \mathrm{D}$ spacetime, as well as in the 5D spacetime. When $A=i$, we have:

$$
T_{; k}^{i k}=f F_{k}^{i} j_{e}^{k}+\rho \alpha^{2} f^{2} \Phi^{-3} \Phi^{, i}+\rho f^{-1} f_{, k} v^{i} v^{k} .
$$

In the coordinate indices, the equation of motion for the pressureless matter can be written as:

$$
\rho v_{, \lambda}^{\mu} v^{\lambda}=-\rho \Gamma_{\lambda \nu}^{\mu} v^{\lambda} v^{\nu}+f F_{\nu}^{\mu} j_{e}^{\nu}+\rho \alpha^{2} f^{2} \Phi^{-3}\left(\Phi^{, \mu}+\Phi_{, \nu} v^{\mu} v^{\nu}\right) .
$$

It is seen that the motion is governed by three forces: gravitational, electromagnetic and scalar field forces. The factor $f$ influences explicitly both the electromagnetic and scalar field forces. The scalar field force is proportional to the scalar field gradient. In addition, the second term of the scalar field force is a new result, which indicates that the scalar field with a gradient exerts more force on moving particles.

For the motion of a single particle, the 5D action of matter can be represented as:

$$
\bar{S}_{m}=-\int m \bar{d} s
$$

According to the principle of variation $\delta \bar{S}_{m}=0$, we have the equation of particle motion in the 5D spacetime as:

$$
\frac{d \bar{u}}{\bar{d} s}+\bar{\Gamma}_{B C}^{A} \bar{u}^{B} \bar{u}^{C}=0
$$

When $A=4$, we have again $\alpha=$ constant. When $A=i$, we have:

$$
\frac{d v^{i}}{d s}+\Gamma_{j k}^{i} v^{i} v^{k}-2 \sqrt{G} \alpha f F_{j}^{i} v^{j}-\alpha^{2} f^{2} \Phi^{-3} \Phi^{\prime i}-v^{i} \frac{d \ln (f)}{d s}=0
$$

In the coordinate indices, the equation of motion for a single particle can be written as:

$$
\frac{d v^{\mu}}{d s}+\Gamma_{\lambda \nu}^{\mu} v^{\lambda} v^{\nu}-2 \sqrt{G} \alpha f F^{\mu \lambda} g_{\lambda \nu} v^{\nu}-\alpha^{2} f^{2} \Phi^{-3} \Phi^{\prime \mu}-v^{\mu} \frac{d \ln (f)}{d s}=0 .
$$

This is similar to Equation (74). Again, the last term shows that the scalar field force may accelerate a particle more significantly if it is moving faster. This may have an important effect in particle physics. 


\section{Static Spherically-Symmetric Field Solution of the 5D Fully-Covariant KK $\Phi$ Theory}

\subsection{Exact External Field Solution}

For a static spherically-symmetric system, we usually write the isotropic line element of the 4D spacetime as [53]:

$$
-d s^{2}=g_{\mu \nu} d x^{\mu} d x^{\nu}=-e^{\nu} d t^{2}+e^{\lambda}\left(d r^{2}+r^{2} d \theta^{2}+r^{2} \sin ^{2} \theta d \phi^{2}\right),
$$

where $\lambda$ and $\nu$ are functions of the radial coordinate $r$. Then, the non-zero components of the Einstein curvature tensor are:

$$
\begin{gathered}
G_{0}^{0}=e^{-\lambda}\left(\lambda^{\prime \prime}+\frac{1}{4} \lambda^{\prime 2}+\frac{2 \lambda^{\prime}}{r}\right), \\
G_{1}^{1}=e^{-\lambda}\left(\frac{1}{4} \lambda^{\prime 2}+\frac{1}{2} \lambda^{\prime} \nu^{\prime}+\frac{\lambda^{\prime}+\nu^{\prime}}{r}\right), \\
G_{2}^{2}=G_{3}^{3}=e^{-\lambda}\left(\frac{1}{2} \nu^{\prime \prime}+\frac{1}{2} \lambda^{\prime \prime}+\frac{1}{4} \nu^{\prime 2}+\frac{\nu^{\prime}+\lambda^{\prime}}{2 r}\right),
\end{gathered}
$$

where the prime ' is the first order derivative with respect to $r$ and the double prime " is the second order derivative with respect to $r$. The electromagnetic field Equation (66) can be easily integrated. The non-zero components of $H^{\mu \nu}$ and $H_{\mu \nu}$ are:

$$
\begin{gathered}
H^{01}=-H^{10}=\frac{Q}{r^{2}} \exp \left[-\frac{\nu+3 \lambda}{2}\right], \\
H_{01}=-H_{10}=-\frac{Q}{r^{2}} \exp \left[\frac{\nu-\lambda}{2}\right] .
\end{gathered}
$$

Here, $Q$ is the total electric charge in the sphere with radius $r$. The gravitation and the scalar field in the 5D spacetime with a pressureless matter are then determined by the following equations:

$$
\begin{aligned}
& e^{-\lambda}\left(\lambda^{\prime \prime}+\frac{1}{4} \lambda^{\prime 2}+\frac{2 \lambda^{\prime}}{r}\right)=8 \pi G f^{-1} \Phi^{-1} T_{0}^{0}(m)+\frac{Q^{2} G}{\Phi^{4} r^{4}} e^{-2 \lambda}+\frac{\Phi^{\prime} \nu^{\prime}}{2 \Phi} e^{-\lambda} \\
& -\frac{8 \pi G}{3} f^{-1} \Phi^{-1} T_{\mu}^{\mu}(m)+\frac{16 \pi G \alpha^{2} \rho}{3 \Phi^{2} \sqrt{\Phi^{2}+\alpha^{2}}}, \\
& e^{-\lambda}\left(\frac{1}{4} \lambda^{\prime 2}+\frac{1}{2} \lambda^{\prime} \nu^{\prime}+\frac{\lambda^{\prime}+\nu^{\prime}}{r}\right)=8 \pi G f^{-1} \Phi^{-1} T_{1}^{1}(m)+\frac{Q^{2} G}{\Phi^{4} r^{4}} e^{-2 \lambda}+ \\
& \frac{1}{\Phi}\left(\Phi^{\prime \prime}-\frac{1}{2} \Phi^{\prime} \lambda^{\prime}\right) e^{-\lambda}-\frac{8 \pi G}{3} f^{-1} \Phi^{-1} T_{\mu}^{\mu}(m)+\frac{16 \pi G \alpha^{2} \rho}{3 \Phi^{2} \sqrt{\Phi^{2}+\alpha^{2}}}, \\
& e^{-\lambda}\left[\frac{\Phi^{\prime \prime}}{\Phi}+\frac{\Phi^{\prime}}{\Phi}\left(\frac{2}{r}+\frac{\lambda^{\prime}+\nu^{\prime}}{2}\right)\right]=-\frac{2 Q^{2} G}{\Phi^{4} r^{4}} e^{-2 \lambda}+\frac{8 \pi G}{3} f^{-1} \Phi^{-1} T_{\mu}^{\mu}(m)-\frac{16 \pi G \alpha^{2} \rho}{3 \Phi^{2} \sqrt{\Phi^{2}+\alpha^{2}}} .
\end{aligned}
$$

To solve the exact external solution, we can let $\rho=0$ (i.e., all $T_{\mu}^{\mu}(m)=0$ with $\left.\mu=0,1,2,3\right)$. Then, the field equations become:

$$
\lambda^{\prime \prime}+\frac{1}{4} \lambda^{\prime 2}+\frac{2 \lambda^{\prime}}{r}=\frac{Q^{2} G}{\Phi^{4} r^{4}} e^{-\lambda}+\frac{\Phi^{\prime} \nu^{\prime}}{2 \Phi},
$$




$$
\begin{gathered}
\frac{1}{4} \lambda^{\prime 2}+\frac{1}{2} \lambda^{\prime} \nu^{\prime}+\frac{\lambda^{\prime}+\nu^{\prime}}{r}=\frac{\Phi^{\prime \prime}}{\Phi}-\frac{\Phi^{\prime} \lambda^{\prime}}{2 \Phi}+\frac{Q^{2} G}{\Phi^{4} r^{4}} e^{-\lambda} \\
\frac{\Phi^{\prime \prime}}{\Phi}+\frac{\Phi^{\prime}}{\Phi}\left(\frac{2}{r}+\frac{\lambda^{\prime}+\nu^{\prime}}{2}\right)=-\frac{2 Q^{2} G}{\Phi^{4} r^{4}} e^{-\lambda} .
\end{gathered}
$$

From [33,41], the exact external solution of the field equations (Equations (85)-(87)) can be represented as:

$$
\begin{gathered}
e^{\lambda}=\left(1-\frac{B^{2}}{r^{2}}\right)^{2} \psi^{-2} \\
e^{\nu}=\psi^{2} \Phi^{-2} \\
\Phi^{2}=a_{1} \psi^{p_{1}}+a_{2} \psi^{p_{2}}
\end{gathered}
$$

where:

$$
\psi=\left(\frac{r-B}{r+B}\right)^{\frac{C}{2 B}}
$$

and the seven constants $\left(K, p_{1}, p_{2}, B, C, a_{1}\right.$ and $\left.a_{2}\right)$ hold the following five relations:

$$
\begin{gathered}
p_{1}=1+\sqrt{1+K}, \\
p_{2}=1-\sqrt{1+K}, \\
K=\frac{4\left(4 B^{2}-C^{2}\right)}{C^{2}}, \\
Q^{2}+a_{1} a_{2} C^{2}(1+K) G^{-1}=0, \\
a_{1}+a_{2}=1 .
\end{gathered}
$$

Here, we have used the asymptotic conditions: $e^{\lambda} \rightarrow 1, e^{\nu} \rightarrow 1$ and $\Phi \rightarrow 1$, at $r \rightarrow \infty$. The five constant relations cannot determine the seven unknown constants. Comparing the exact field external solution at the large radial distance with a weak field internal solution, we can have two more constant relations, so we can completely determine all of the constants. In the following section, we first solve the field Equations (85)-(87) of the 5D fully-covariant $\mathrm{KK} \Phi$ theory to have a weak field internal solution.

\subsection{Weak Field Internal Solution}

For weak fields, we can write $e^{\lambda}, e^{\nu}$ and $\Phi$ as:

$$
\begin{aligned}
& e^{\lambda}=1+\delta \lambda, \\
& e^{\nu}=1+\delta \nu, \\
& \Phi=1+\delta \Phi .
\end{aligned}
$$

Substituting Equations (100)-(102) into the field equation Equations (85)-(87) and keeping the first order, we have the weak field equations:

$$
\frac{1}{r^{2}} \frac{d}{d r}\left[r^{2}(\delta \lambda)^{\prime}\right]=-\frac{16 \pi G \rho}{3 \sqrt{1+\alpha^{2}}},
$$




$$
\begin{gathered}
\frac{1}{r^{2}} \frac{d}{d r}\left[r^{2}(\delta \Phi)^{\prime}\right]=-\frac{8 \pi G \rho\left(1+3 \alpha^{2}\right)}{3 \sqrt{1+\alpha^{2}}}, \\
\frac{1}{r} \frac{d}{d r}(\delta \nu+\delta \lambda+2 \delta \Phi)=0 .
\end{gathered}
$$

Here, we have not assumed the density of matter $\rho$ nor the energy-momentum tensors to be zero, because we are solving the internal field. Integrating and using the condition $\delta \lambda \rightarrow 0, \delta \nu \rightarrow 0$ and $\delta \Phi \rightarrow 0$, as $r \rightarrow \infty$, we have:

$$
\begin{gathered}
\delta \lambda=\frac{4 G m}{3 \sqrt{1+\alpha^{2}}} \frac{1}{r}, \\
\delta \nu=-\frac{4 G m\left(2+3 \alpha^{2}\right.}{3 \sqrt{1+\alpha^{2}}} \frac{1}{r}, \\
\delta \Phi=\frac{2 G m\left(1+3 \alpha^{2}\right)}{3 \sqrt{1+\alpha^{2}}} \frac{1}{r} .
\end{gathered}
$$

Then, the internal solution of the weak fields is obtained as:

$$
\begin{gathered}
H^{01}=-H^{10}=-H_{01}=H_{10}=\frac{Q}{r^{2}}, \\
e^{\lambda}=1+\frac{4 G m}{3 \sqrt{1+\alpha^{2}}} \frac{1}{r} \\
e^{\nu}=1-\frac{4 G m\left(2+3 \alpha^{2}\right)}{3 \sqrt{1+\alpha^{2}}} \frac{1}{r} \\
\Phi=1+\frac{2 G m\left(1+3 \alpha^{2}\right)}{3 \sqrt{1+\alpha^{2}}} \frac{1}{r}
\end{gathered}
$$

\subsection{Determination of the Exact Solution Constants}

The exact field external solution of the 5D fully-covariant $\mathrm{KK} \Phi$ theory includes two independent constants. The five constant relation Equations (95)-(99) cannot determine the seven unknown constants $\left(K, p_{1}, p_{2}, B, C, a_{1}, a_{2}\right)$ in the exact solution.

In Einstein's general theory of relativity, one can get the Schwarzschild solution of a spherically-symmetric object with mass $M$ without any undetermined parameter, except for the mass $M$, by the following three equivalent methods. The first is the Newton gravitational potential method, which operates in two steps. We first obtain an exact external solution with one integration constant and then compare the obtained exact solution with the Newton gravitational potential to determine the constant $[53,54]$. The second is the volume integration method. For a pressureless or ideal fluid matter, we can complete a volume integration to the non-vacuum Einstein field equation over a sphere to directly find the Schwarzschild solution without any unknown parameter. The third method is the weak field internal solution method. Furthermore, for a pressureless matter, we first obtain a weak field interior solution (it is the same as that given by the Newton gravitational potential), as well as an exact external solution, and then compare them on the surface of a sphere with a sufficiently large radius to determine the integration constant of the exact external solution. These three methods lead to the same result: the Schwarzschild solution. 
In a $5 \mathrm{D}$ KK $\Phi$ theory, however, there are three types of fields (gravitational, electromagnetic and scalar fields), and also, the field equations are highly nonlinear. Thus, the first and second methods described above are not appropriate for the 5D KK $\Phi$ theory. The third (or the weak field internal solution) method may be applicable to the 5D KK $\Phi$ theory. It is important to find the weak field interior solution when we apply this method to the 5D KK $\Phi$ theory. Chodos and Detweiler [33] determined the independent constants by using the weak field limit at a large distance in the same way as in the Schwarzschild solution; the single constant was determined by using the Newton potential of weak gravity [53,54]. To find the weak field internal solution, Chodos and Detweiler assumed the fifteenth component of the 5D energy-momentum tensor of matter to be $\bar{T}^{44}=s \rho$, where $s$ is the scalar charge per unit mass and $\rho$ is the matter density. Since they introduced an unknown parameter, the new results obtained from the 5D KK $\Phi$ theory were non-conclusive. In [33], a weak field interior solution of the 5D KK $\Phi$ theory was obtained, but it includes an unknown parameter named the scalar charge. The reason is because they introduced the artificial parameter to describe the 5D pressureless matter. In the 5D fully-covariant KK $\Phi$ theory, the author derived $\bar{T}^{44}$ (Equation (61)) without the scalar charge. With this weak field internal solution method, we should be able to determine all constants in the exact field external solution.

Expanding the exact external solution of the 5D fully-covariant $\mathrm{KK} \Phi$ theory and comparing it with the weak field interior solution on a spherical surface at a large distance, we obtain two more independent constant relations, which can be used along with the five constant relations to determine all of the seven unknown constants,

$$
\begin{gathered}
C=\frac{2 G m}{3 \sqrt{1+\alpha^{2}}}, \\
-C\left(a_{1} p_{1}+a_{2} p_{2}\right)=\frac{4 G m\left(1+3 \alpha^{2}\right)}{3 \sqrt{1+\alpha^{2}}} .
\end{gathered}
$$

Coupling Equations (113) and (114) with the other constant relation Equations (95)-(99), we can exactly solve all seven unknown constants in the exact external solution as:

$$
\begin{gathered}
K=8, \\
p_{1}=4, \quad p_{2}=-2, \\
a_{1}=-\alpha^{2}, \quad a_{2}=1+\alpha^{2}, \\
C=\frac{2 G m}{3 \sqrt{1+\alpha^{2}}}, \quad B=\frac{G m}{\sqrt{3\left(1+\alpha^{2}\right)}} .
\end{gathered}
$$

The cgs unit system with $c=1$ is adapted in this study. If the light speed $c$ is retained, the constants $B$ and $C$ need to be multiplied by $1 / c^{2}$. This set of constants is the simplest and most elegant, because of $K=8$, for the field solution of the $5 \mathrm{D}$ fully-covariant $\mathrm{KK} \Phi$ theory to be non-trivial. It is seen that the obtained static, spherically-symmetric field external solution of the $5 \mathrm{D}$ fully-covariant $\mathrm{KK} \Phi$ theory does not involve any unknown parameter and undetermined constant. The exact external solution is completely determined by giving the gravitational constant $G$, the mass $M$ and the electric charge $Q$ or $\alpha$. Therefore, the radial dependences of the most general time-independent, spherically-symmetric field external solution is completely determined when the charge and mass of matter are given. In the following section, we will analyze the solution properties and their astrophysical applications. 


\section{Field Solution Properties and Astrophysical Applications}

\subsection{Solution Properties}

To see the field solution properties, we plot $e^{\lambda}, e^{\nu}, \Phi$ and $E / E_{c}$ in Figure 1 as functions of the normalized radial distance $r / B$ with five different charge-to-mass ratios $\alpha=0,1,10,100,1,000$. Here, $E$ is the electric field strength defined by $E=F_{10}$ and $E_{c}$ is the Coulomb electric field strength, $E_{c}=Q / r^{2}$. It is seen that all of these variables of the field solution asymptotically approach unity when $r$ is sufficiently large or approaches infinity. When $r$ tends to be small, the solution $e^{\lambda}$, which is independent of $\alpha$ at any normalized radial distance, reaches a maximum of about two at $r \simeq 2 B$ and then wanes to zero rapidly as $r \rightarrow B$ (Figure 1a). The solutions, $e^{\nu}, \Phi$ and $E / E_{c}$, however, are significantly affected by the electric charge of the matter when $r$ is not sufficient large. Especially, as $r \rightarrow B$, both $e^{\nu}$ and $E / E_{c}$ become zero (Figure $1 \mathrm{~b}, \mathrm{~d}$ ), while $\Phi$ tends to infinity (Figure 1c). For a non-massive or non-dense object, the effects of electric charge and scalar field on the gravitational, electromagnetic and scalar fields are very small, no matter how big the charge-to-mass ratio $\alpha$ is, because $B$ is very small in comparison with the size of the object.
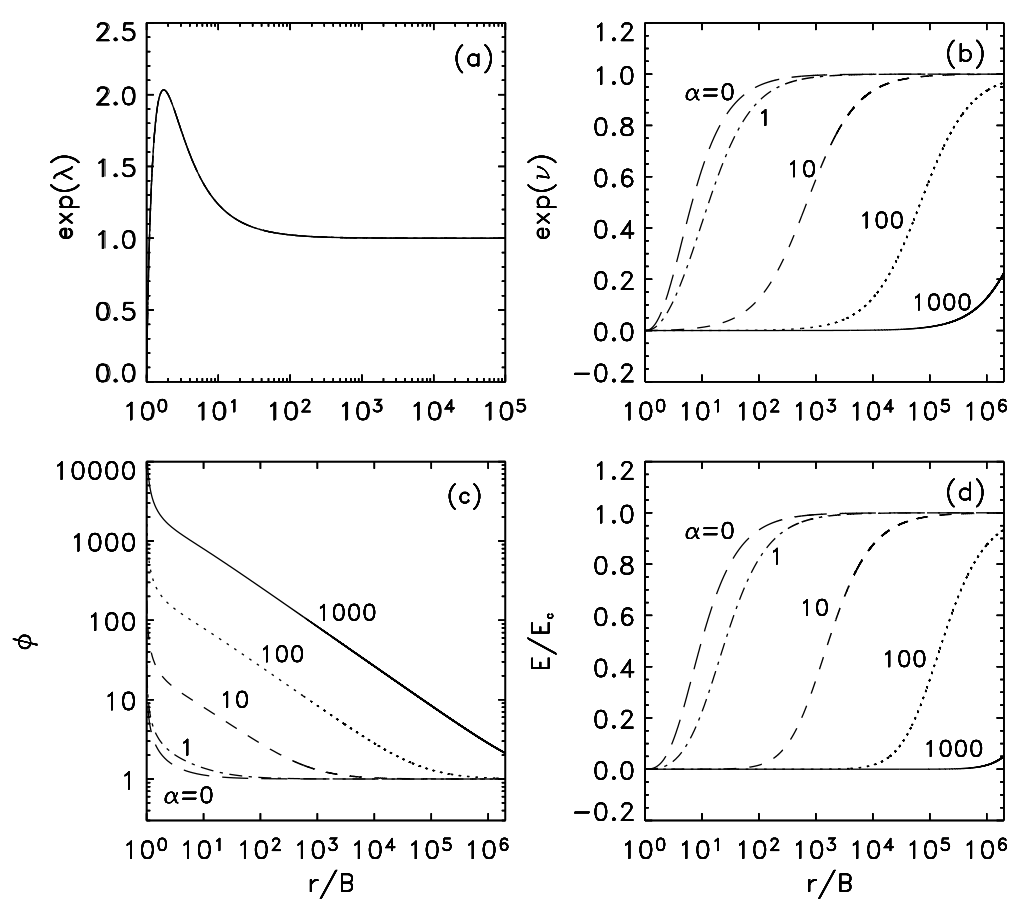

Figure 1. Radial dependences of the static spherically-symmetric solutions of gravitation, electromagnetism and the scalar field with $\alpha=0,1,10,100,1,000$ (taken from [45]). (a) $e^{\lambda}$ vs. $r / B$; (b) $e^{\nu}$ vs. $r / B$; (c) $\Phi$ vs. $r / B$; and (d) $E / E_{c}$ vs. $r / B$.

Table 1 shows that the values of $B$ for a spherical object with different $\alpha$ and $m$. It is seen that $B$ increases if either $m$ increases or $\alpha$ decreases. In comparison with the general theory of relativity, $B$ is significantly smaller than the Schwarzschild radius, especially when the spherical body is highly charged. Therefore, the electric charge effects on the fields are negligible if the charged body is not dense and massive. 
Table 1. Values of $B$ in centimeters with different $\alpha$ and $m$ in grams.

\begin{tabular}{lllllll}
\hline & $\boldsymbol{m}=\mathbf{1 0}^{-\mathbf{2 4}}$ & $\boldsymbol{m}=\mathbf{1 0}^{-\mathbf{1 0}}$ & $\boldsymbol{m}=\mathbf{1}$ & $\boldsymbol{m}=\mathbf{1 0}^{\mathbf{1 0}}$ & $\boldsymbol{m}=\mathbf{1 0}^{\mathbf{3 3}}$ & $\boldsymbol{m}=\mathbf{1 0}^{\mathbf{4 0}}$ \\
\hline$\alpha=10^{18}$ & $4 \times 10^{-71}$ & $4 \times 10^{-57}$ & $4 \times 10^{-47}$ & $4 \times 10^{-37}$ & $4 \times 10^{-14}$ & $4 \times 10^{-7}$ \\
$\alpha=10^{10}$ & $4 \times 10^{-63}$ & $4 \times 10^{-49}$ & $4 \times 10^{-39}$ & $4 \times 10^{-29}$ & $4 \times 10^{-6}$ & $4 \times 10^{1}$ \\
$\alpha=10^{5}$ & $4 \times 10^{-58}$ & $4 \times 10^{-44}$ & $4 \times 10^{-34}$ & $4 \times 10^{-24}$ & $4 \times 10^{-1}$ & $4 \times 10^{6}$ \\
$\alpha=10^{2}$ & $4 \times 10^{-55}$ & $4 \times 10^{-41}$ & $4 \times 10^{-31}$ & $4 \times 10^{-21}$ & $4 \times 10^{2}$ & $4 \times 10^{9}$ \\
$\alpha=1$ & $3 \times 10^{-53}$ & $3 \times 10^{-39}$ & $3 \times 10^{-29}$ & $3 \times 10^{-19}$ & $3 \times 10^{4}$ & $3 \times 10^{11}$ \\
$\alpha=0$ & $4 \times 10^{-53}$ & $4 \times 10^{-39}$ & $4 \times 10^{-29}$ & $4 \times 10^{-19}$ & $4 \times 10^{4}$ & $4 \times 10^{11}$ \\
\hline
\end{tabular}

Figure 2 plots the field solutions $e^{\lambda}, e^{\nu}, \Phi$ and $E / E_{c}$ of a star with 1.5 solar masses and $\alpha=0,1$, $10,100,1,000$ as functions of the radial distance $r$ in meters. It is seen that all of the field solutions are dependent on $\alpha$ and approach unity when $r$ tends to infinity. The solution $e^{\lambda}$ has the same profile, but the peak shifts towards the smaller radius end as $\alpha$ increases (Figure 2a). When $r \rightarrow B$, all functions $e^{\lambda}, e^{\nu}$ and $E / E_{c}$ approach zero (Figure 2a,b,d), while $\Phi$ approaches infinity (Figure 2c). A star, if it is compact, has a large scalar field, but a weak gravitational field in comparison with the Newton gravitational field and a weak electric field in comparison with the Coulomb electric field. These derivations from the Newton gravitational law and Coulomb electric law are because the equivalent gravitational constant is significantly decreased (or shed) and the space is greatly polarized by the strong scalar field (see the following Sections 6.4 and 6.6).
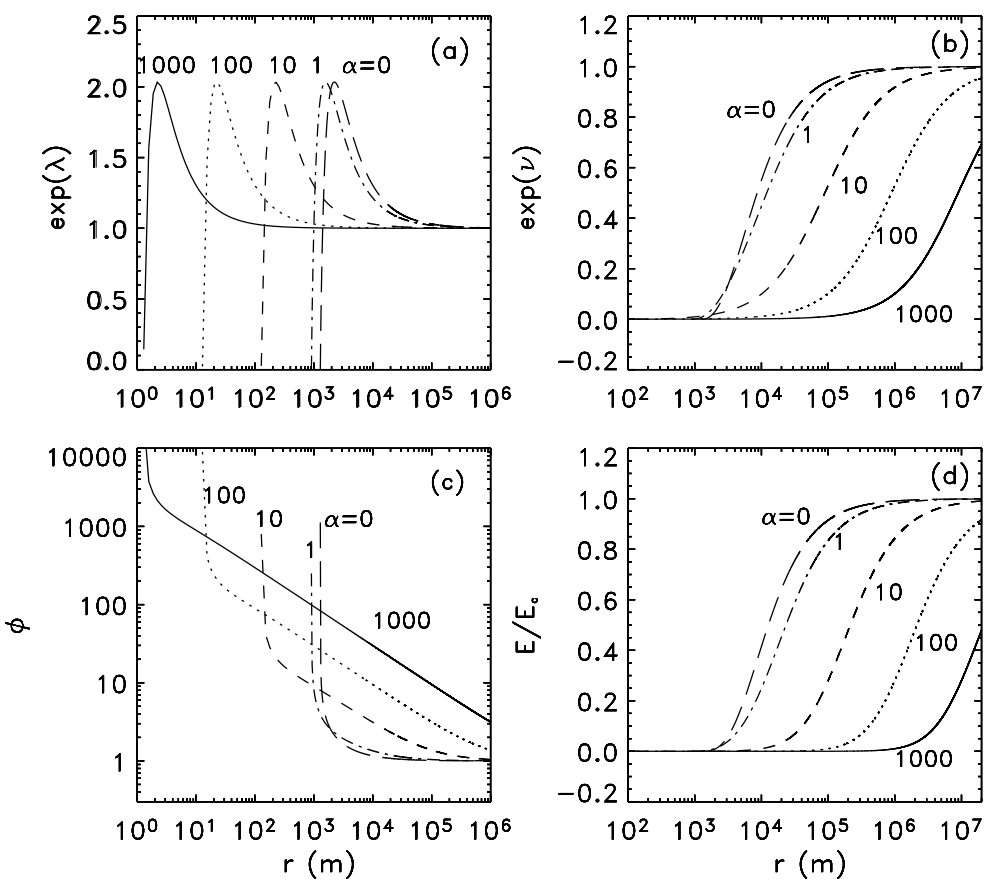

Figure 2. Radial dependences of the static, spherically-symmetric solutions of gravitation, electromagnetism and the scalar field for a star with 1.5 solar masses and $\alpha=$ $0,1,10,100,1,000$. (a) $e^{\lambda} v s . r$; (b) $e^{\nu} v$ s. $r$; (c) $\Phi$ vs. $r$; and (d) $E / E_{c} v$ s. $r$. 


\subsection{Field Solution in the Einstein Frame and Fundamental Tests}

The 4D metric solution obtained above interprets the 4D spacetime in a frame, usually called the Jordan frame. It can be transformed into the Einstein frame [55] via the following conformal transformation,

$$
d s_{E}^{2}=\phi d s^{2}
$$

Mathematically, both the Jordan and Einstein frames are equivalent. Physically, they should also be equivalent if we are able to adjust our time and length measuring clocks and rods to be independent of theory [56]. However, if we fix our measuring clocks and rods in one frame, then the two frames are physically nonequivalent. Determining the planet perihelion procession, the deflection of light by the Sun, the redshift of the Sun's light and the delay of radar echoes (the four fundamental tests of general relativity) in both frames and comparing with the results of experimental measurements can tell us on which frame we are standing.

The 00- and 11-components of the 4D spacetime metric in the Einstein frame are:

$$
\begin{gathered}
-g_{00}^{E}=\phi e^{\nu}=\frac{\psi^{2}}{\phi}, \\
g_{11}^{E}=\phi e^{\lambda}=\left(1-\frac{B^{2}}{r^{2}}\right)^{2} \frac{\phi}{\psi^{2}} .
\end{gathered}
$$

In the weak field approximation (i.e., $B<<r$ or, in other words, the gravitational potential energy of a particle is much less than its rest energy), we have:

$$
\begin{gathered}
-g_{00}^{E}=1-\frac{2 G m \sqrt{1+\alpha^{2}}}{r} \longrightarrow 1-\frac{2 G m}{r}, \quad \text { if } \alpha^{2}<<1 \text { or } 0, \\
g_{11}^{E}=1+\frac{2 G m \sqrt{1+\alpha^{2}}}{r} \longrightarrow 1+\frac{2 G m}{r}, \quad \text { if } \alpha^{2}<<1 \text { or } 0 .
\end{gathered}
$$

It is seen that for an electrically neutral matter $\alpha^{2}=0$ or weakly charged matter $\alpha<<1$, the field solution of the 5D fully-covariant $\mathrm{KK} \Phi$ theory reduces to the Schwarzschild solution. Considering the normal star that can be electrically charged at most by about 100 Coulomb due to the radiation pressure, we have $\alpha=10^{-20} \sim 0$ for the Sun. Therefore, the four fundamental measurements that have tested Einstein's general theory of relativity have also tested the 5D fully-covariant $K K \Phi$ theory.

To see also the solution properties and differences in the strong field case, we plot in the left column of Figure 3 the field solutions $g_{11}$ and $-g_{00}$ in the Einstein frame as functions of the normalized radial distance $r / B$ with the five different charge-to-mass ratios $\alpha=0,1,10,100,1,000$. It is seen that as $r \rightarrow B$, the 11-component of the metric solution increases and then suddenly decreases to zero. For a greater $\alpha$, the peak $g_{11}$ is higher. The 00-component approaches zero as $r \rightarrow B$. For a compact (or strong field) object (especially when the object is electrically charged), the gravitational field solution of the 5D fully-covariant KK $\Phi$ theory in both the Jordan and Einstein frames significantly deviates from the Schwarzschild solution. To see the difference more quantitatively between them, we plot $-g_{00}$ and $g_{11}$ in both the Jordan and Einstein frames with $\alpha=0$ along the Schwarzschild solution as functions of the radial distance in the right column of Figure 3. 

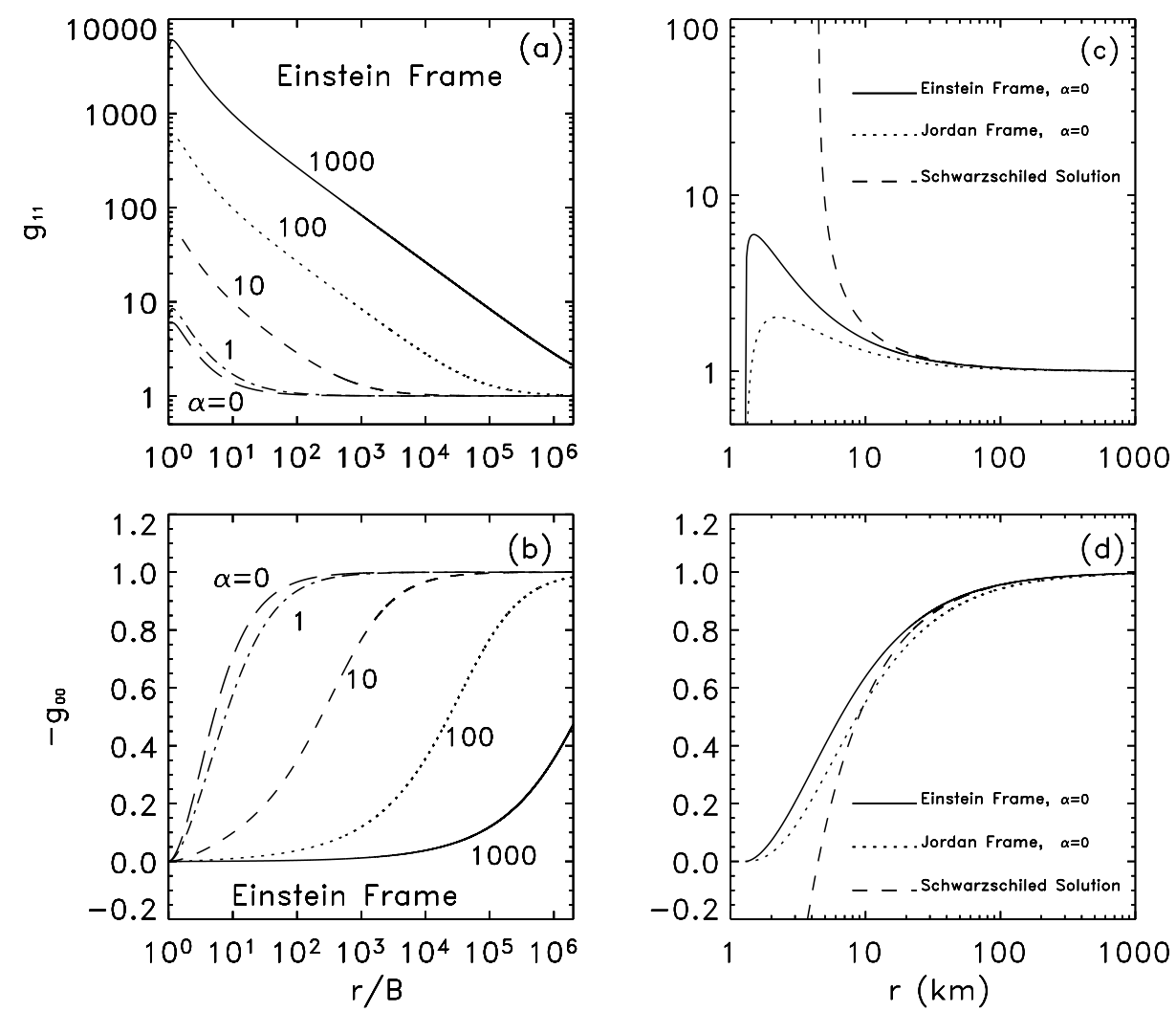

Figure 3. Radial dependences of the static spherically-symmetric KK solution of the gravitational field in the Einstein frame with $\alpha=0,1,10,100,1,000$. (a) $g_{11} v s . r / B$; (b) $-g_{00}$ vs. $r / B$; (c) $g_{11}$ and (d) $-g_{00}$ from the Kaluza-Klein (KK) solution in both Jordan and Einstein frames with that from the Schwarzschild solution for a neutron object with 1.5 solar masses.

\subsection{Electric Redshift and Quasars}

The redshift mechanisms that are well-developed so far include: (1) the Doppler redshift due to motion; (2) the Einstein gravitational redshift due to gravity; and (3) the cosmological redshift due to the expansion of the universe. Recently, the author developed a new redshift mechanism, called electric redshift [45], which is due to electric charge, according to the 5D fully-covariant $K K \Phi$ theory.

In the 5D fully-covariant $\mathrm{KK} \Phi$ theory, light travels on null geodesics, i.e.,

$$
\overline{d s}^{2}=f^{2} d s^{2}=0
$$

Then, along a radial light path, we have,

$$
\sqrt{-g_{00}} d t=\sqrt{g_{11}} d r
$$

It follows that:

$$
\int_{t_{e}}^{t_{o}} \sqrt{-g_{00}} d t=\int_{t_{e}+\delta t_{e}}^{t_{o}+\delta t_{o}} \sqrt{-g_{00}} d t=\int_{r_{e}}^{r_{o}} \sqrt{g_{11}} d r
$$

where $t_{e}$ is the time for the emission of the light and $t_{o}$ is the time for the observation of the light. Since $\delta t_{e, o}=1 / \nu_{e, o}=\lambda_{e, o} / c$, the redshift $Z$ can be determined as:

$$
1+Z=\frac{\lambda_{o}}{\lambda_{e}}=\frac{\nu_{e}}{\nu_{o}}=\frac{\delta t_{o}}{\delta t_{e}}=\frac{\sqrt{-g_{00}\left(r_{e}\right)}}{\sqrt{-g_{00}\left(r_{o}\right)}} .
$$


Here, $\lambda_{e}$ and $\nu_{e}$ (or $\lambda_{o}$ and $\nu_{o}$ ) are the wavelength and frequency of emitted (or observed) light, respectively. Light from a source object is redshifted, because the time interval is increased or the energy of photons is decreased due to the gravitational, electromagnetic and scalar fields. For light emitted from the surface of an object with radius $R$ and observed by a distant observer $\left(r_{e}=R\right.$ and $\left.r_{o}=\infty\right)$, we have:

$$
Z=\sqrt{-g_{00}(R)}-1=e^{-\nu(R) / 2}-1
$$

As shown by this equation, this type of redshift depends on both mass $m$ and electric charge $Q$ and can be called electric-gravitational redshift. The extra part due to electric charge was called electric redshift [45], which can be significant and dominant when the charge-mass ratio is much greater than unity, $\alpha>>1$.

Figure 4a plots $Z$ as a function of $R / B$ with $\alpha=0,1,10,100,1,000$. It is seen that the redshift increases with increasing $\alpha$, but decreases with increasing $R / B$. If $\alpha>10$ and $R<1000 B$, the redshift can be greater than unity. If $\alpha<1$, the redshift cannot be greater than unity, except for $R<3 B$.
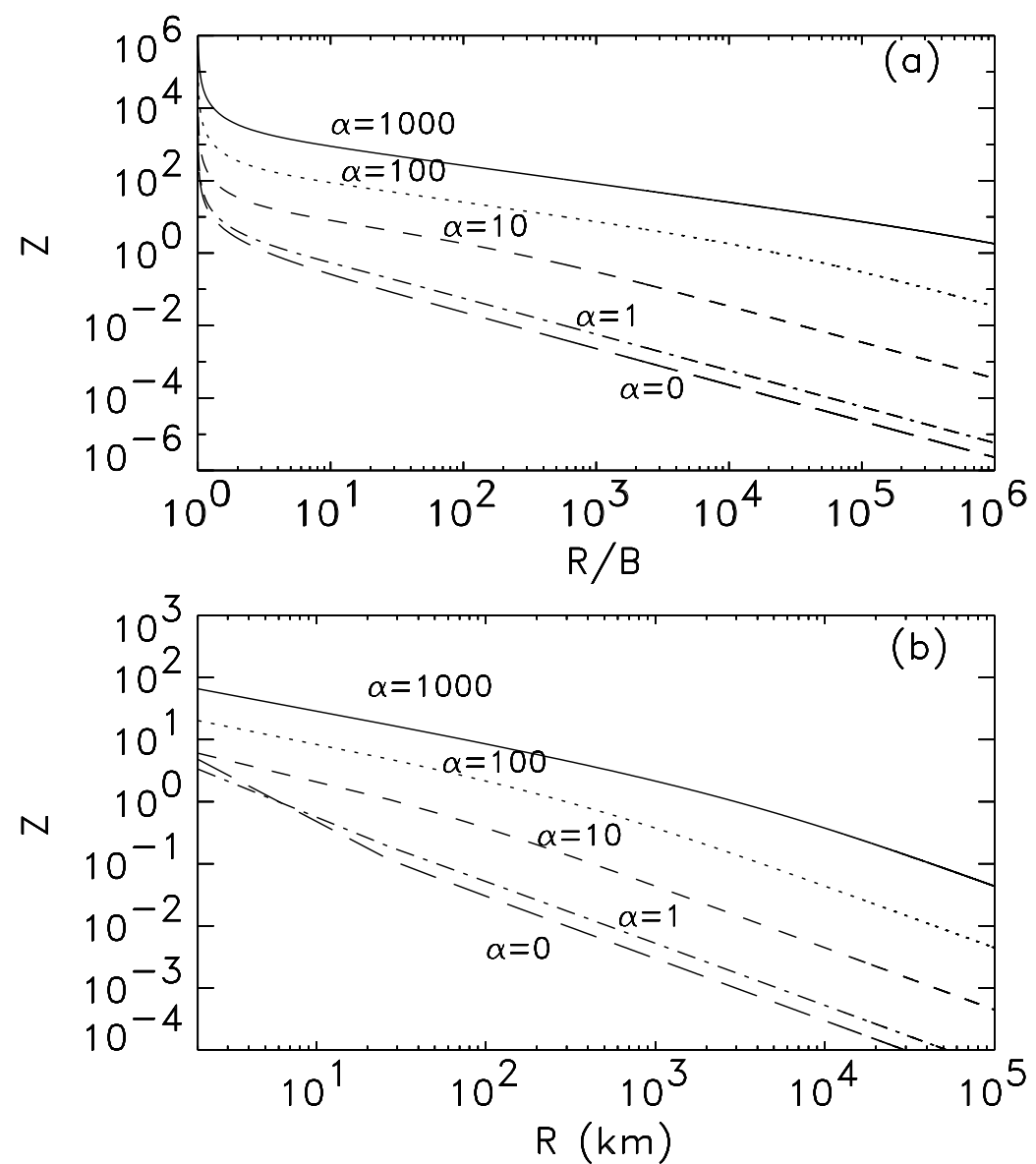

Figure 4. Electric redshifts (taken from [45]). (a) $Z v s$. $R / B$ of a sphere with $\alpha=0,1,10$, 100, 1,000; (b) $Z$ vs. $R$ of a star with 1.5 solar masses and $\alpha=0,1,10,100,1,000$.

To satisfy $R<1,000 B$, the spherical body must be very dense and massive, like a neutron star. Figure $4 \mathrm{~b}$ plots the redshift $Z$ as a function of the radius $R$ of a star with 1.5 solar masses and $\alpha=0,1$, $10,100,1,000$. It is seen that the redshift can exceed unity if $\alpha>10$ and $R<100(\mathrm{~km})$. For the redshift to be greater than unity at $\alpha<1$, the star radius must be less than about $5 \mathrm{~km}$. This result may have a 
great impact on the understanding of quasars with extremely large redshifts. If a quasar is a star as dense as a neutron star, but electrically charged up to a certain amount, the light rays emitted from its surface can be significantly shifted toward the red up to the order of quasar redshift measurements. For instance, considering a star as dense as a neutron star with 1.5 solar masses (in which there are $\sim 2 \times 10^{57}$ neutrons) and radius $R=20(\mathrm{~km})$, the redshift can be as high as $Z \simeq 5$, if $10^{-16} \%$ of neutrons are replaced by protons $\left(\sim 2 \times 10^{41}\right.$ protons or $\left.\alpha \simeq 60\right)$.

It might be possible that a star as dense and massive as a neutron star is charged due to holding a certain amount of net protons or nuclei. The fraction of protons in neutron stars and the effect of electric charge in compact stars have been considered for years [57,58]. To reproduce the observations of Geminga, a model of a dense neutron star with localized protons was proposed $[59,60]$. The electric redshift mechanism proposed in [45] may help people to understand more about quasars. If a quasar is a dense, massive and highly charged star as a result of holding extra protons, then not only the evidence of higher redshift quasars in association with lower redshift galaxies, but also the luminosity variations on short timescales and the existence of a strong magnet moment can be understood [61-63]. In addition, the quasi-stellar characteristic of a quasar is also understandable, because a dense, massive and highly charged star should be surrounded by a hot, dense, thick, electron-rich plasma cloud or sheath. This new redshift mechanism does not have to go against the Big Bang cosmology, because the electric redshifts are negligible for normal stars, galaxies and large-scale matters.

In the Einstein frame, the electric redshift is given by:

$$
Z^{E} \simeq \frac{1}{\sqrt{\phi}} e^{-\nu(R) / 2}-1
$$

It is relatively weak in comparison with that in the Jordan frame, but still much more significant than the gravitational redshift when the object is compact and highly charged electrically [64].

\subsection{Gravitational Field Shielding and Supernova Explosions}

In the $5 \mathrm{D}$ fully-covariant $\mathrm{KK} \Phi$ theory of the Einstein frame, the gravitational field of a spherical object can be obtained from the equation of motion (Equation (74) or Equation (78)) for neutral matter or particles as: [46]

$$
g=\frac{c^{2}}{2 \phi^{2}}\left(\frac{d \phi}{d r}+\phi \frac{d \nu}{d r}\right) e^{-\lambda} .
$$

The Newton gravitational field is given by $g^{N}=G M / r^{2}$. Figure 5 plots the KK (solid line) and Newton (dotted-dashed line) gravitational fields as functions of $r$ for a neutral object (i.e., $\alpha=0$ ) with 1.5 solar masses. It is seen that when the object shrinks to about $B$ in radius (i.e., $r \rightarrow B$ ), the KK gravitational field suddenly goes to zero (turns off or is shed). The radius difference $\delta r$ for the gravitational field turning off or shielding is only a few tens of meters, which is much smaller than $B$. For the case of Figure 5 (i.e., an object with 1.5 solar masses), $\delta r \sim 20 \mathrm{~m}$ and $B \sim 1.3 \mathrm{~km}$. The gravitational field of the compact object, when its mass-radius ratio is equal to $M / B=\sqrt{3} c^{2} / G \sim 2.3 \times 10^{27} \mathrm{~kg} / \mathrm{m}$, is shed by the strong scalar field or the huge mass enclosed. 


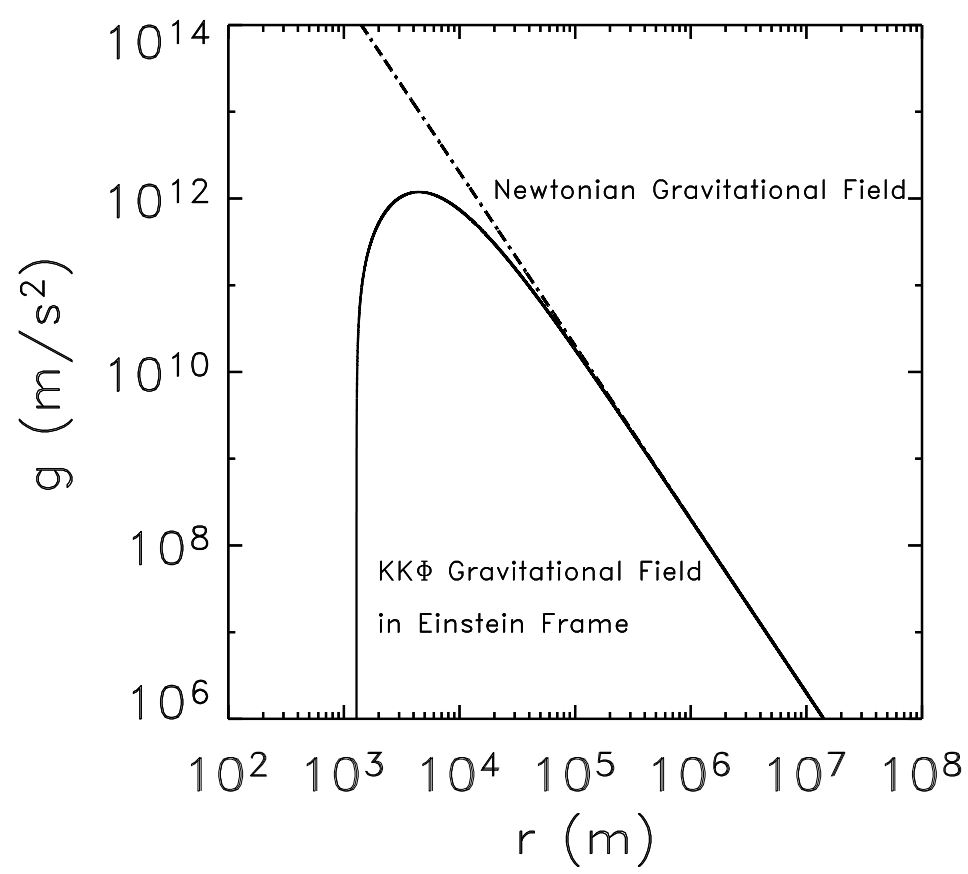

Figure 5. KK (solid line) and Newtonian (dot-dashed line) gravitational fields at the surface of a spherical object with 1.5 solar masses $v s$. the radius of the object [46].

According to the 5D fully-covariant $\mathrm{KK} \Phi$ theory, the gravitational and electromagnetic fields can be significantly reduced by the scalar field. The decrease of the electromagnetic field by the scalar field was called vacuum polarization [12,13]. We call the decrease of the gravitational field by the scalar field gravitational field shielding or spacetime flattening by the scalar field $[46,65]$. As shown in our previous studies, a massive compact object can produce a significant scalar field, which reduces the gravitational field (or varies the equivalent gravitational constant) around the object. The scalar field not only polarizes the space by reducing or shielding the electromagnetic field, but also flattens the spacetime by reducing or shielding the gravitational field.

The critical density of the core for gravitational field shielding is given by:

$$
\rho_{c}=\frac{3 M}{4 \pi B^{3}}=\frac{9 \sqrt{3} c^{6}}{4 \pi G^{3} M^{2}}
$$

where the light speed is retained and $M$ is the core mass. It is seen that the critical density is inversely proportional to the square of the core mass.

When the gravity is shed, the large pressure causes the core to expand and throw the mantle material of the supernova outward. The energy release for the supernova explosion can be roughly estimated according to the work done by the thermal pressure to expand the core from $B$ to $B+\delta r$ :

$$
E \sim \int P_{i} d V=4 \pi \int_{B}^{B+\delta r} P_{i} r^{2} d r
$$

where $P_{i}$ is the thermal pressure given by $P_{i}=(3 / 2) n k T$ with $n$ being the baryon number density of the core, $k$ the Boltzmann constant and $T$ the temperature. Because $\delta r<<B$, the thermal pressure can be considered as a constant in this expansion. Considering that a neutron star may have a temperature as high as a hundred to a thousand billion degrees (e.g., $5 \times 10^{11} \mathrm{~K}$ ) at the moment of its birth by an 
explosion of a supernova [66], we can choose $T \sim 5 \times 10^{11} \mathrm{~K}$. Substituting $P_{i}$ into Equation (132), we have:

$$
E=4 \pi P_{i} B^{2} \delta r=6 \pi n k T B^{2} \delta r=\frac{9 M}{2 m_{p}} k T \frac{\delta r}{B}=\frac{9 \sqrt{3} c^{2} k T}{2 G m_{p}} \delta r .
$$

For $\delta r=20 \mathrm{~m}$ and $T=5 \times 10^{11} \mathrm{~K}$, we have $E \sim 8 \times 10^{44} \mathrm{~J}$, which is about the order of supernova explosion energy.

In the above calculation, the thermal pressure of ideal gas is applied. At $T=5 \times 10^{11} \mathrm{~K}$, the thermal pressure is $P_{i}=(3 / 2) n k T \simeq 2 \times 10^{36} \mathrm{~Pa}$. If we consider the core matter as a degenerate gas, the pressure at the critical density will be many orders of magnitude higher (e.g., $10^{40} \mathrm{~Pa}[67,68]$ ), which crucially depends on the equation of state (EOS) of the core matter. With this consideration of degeneracy, the work done by the pressure in the expansion of the core when the gravitational field turns off will be the order of $10^{48} \mathrm{~J}$. It should be noted that the gravity will partially come back or turn on as the core expands. The gravity resumed will dissipate the energy transformation from the expansion of the core to the explosion of the mantle material. Ten-thousandths of the total work done by the pressure when the gravitational field shielding takes place can provide enough energy to ignite the supernova explosion. To characterize the explosion, a more realistic EOS must be considered.

\subsection{Singularity and Gravitationless Black Hole}

The obtained field solution of the 5D covariant $\mathrm{KK} \Phi$ theory is singular at the point of $r=B$. The functions, $e^{\lambda}, e^{\nu}$ and $F_{10}$, are limited to zero, but $\Phi$ approaches infinity at this point. All of the functions are undefined and, hence, not physical when $r<B$, due to having a number, such as $(-1)^{1 / \sqrt{3}}$, without a definition in mathematics. Thus, the field solution is only valid for $r \geq B$ (or $\psi \leq 1$ ). We cannot transform topologically from the space with $r \geq B$ into that with $r<B$. Therefore, the field solution of the 5D fully-covariant $\mathrm{KK} \Phi$ theory is singular at $r=B$. An object with a radius equal to or less than $B$ can be considered as a black hole. Given the same mass, the KK black hole has a smaller size than the Schwarzschild black hole, by a factor of $\sqrt{3} / 6$ in radius.

For a star core with radius $R$ and mass $M$ (so that the core density $\rho=3 M /\left(4 \pi R^{3}\right)$ ), its gravitational pressure can be obtained by [47]:

$$
P_{g}=-\int_{0}^{R} \rho g d r
$$

where $g$ is given by Equation (130). When the core collapses into a black hole, the work done by the gravitational field can be calculated by:

$$
W_{g}=4 \pi \int_{R_{c}}^{B} P_{g} R^{2} d R
$$

where $R_{c}$ is the core radius at initial $\left(R_{c}>>B\right)$. On the other hand, the core gas pressure works against the gravitational collapse. The work done by the core gas pressure $P_{i}$ can be calculated by:

$$
W_{i}=-4 \pi \int_{R_{c}}^{B} P_{i} R^{2} d R .
$$

Considering the core matter as a degenerate neutron gas, we can determine $P_{i}$ by:

$$
P_{i}=\frac{1}{8}\left(\frac{3 c^{3} h^{3}}{\pi}\right)^{1 / 3} n_{i}^{4 / 3}
$$


for relativistic and:

$$
P_{i}=\frac{1}{10 m_{n}}\left(\frac{3 h^{3}}{8 \pi}\right)^{2 / 3} n_{i}^{5 / 3}
$$

for non-relativistic, where $m_{n}$ is the neutron mass, $h$ is the Planck constant and $n_{i}$ is the neutron number density.

Integrating Equations (135) and (136) with Equations (134), (137) and (138), we plot in Figure 6 the work done by the gravitational field (solid line) and by the pressure of relativistic (dotted line) and non-relativistic (dashed line) degenerate neutron gases as functions of the core mass. It is seen that to collapse into a gravitationless black hole, a relativistic degenerate neutron core must have a mass greater than $\sim 7.5$ solar masses (called the upper limit), while a non-relativistic core need only exceed $\sim 2.7$ solar masses (called the lower limit). A sufficiently cooled neutron star, because the degenerate neutron gas is non-relativistic, will collapse into a gravitationless black hole when it accretes enough matter from a companion star, such that the total mass exceeds the lower limit. A collapsing core of a massive star at the end of evolution can collapse directly into a gravitationless black hole if the core degenerate gas is relativistic and the core mass exceeds the upper limit. If the core degenerate gas is intermediate relativistic, the upper mass limit will be less than 7.5 solar masses. In the work integrations, we have chosen $R_{c} / B=20$, on which the lower and upper mass limits depend, but not significantly.

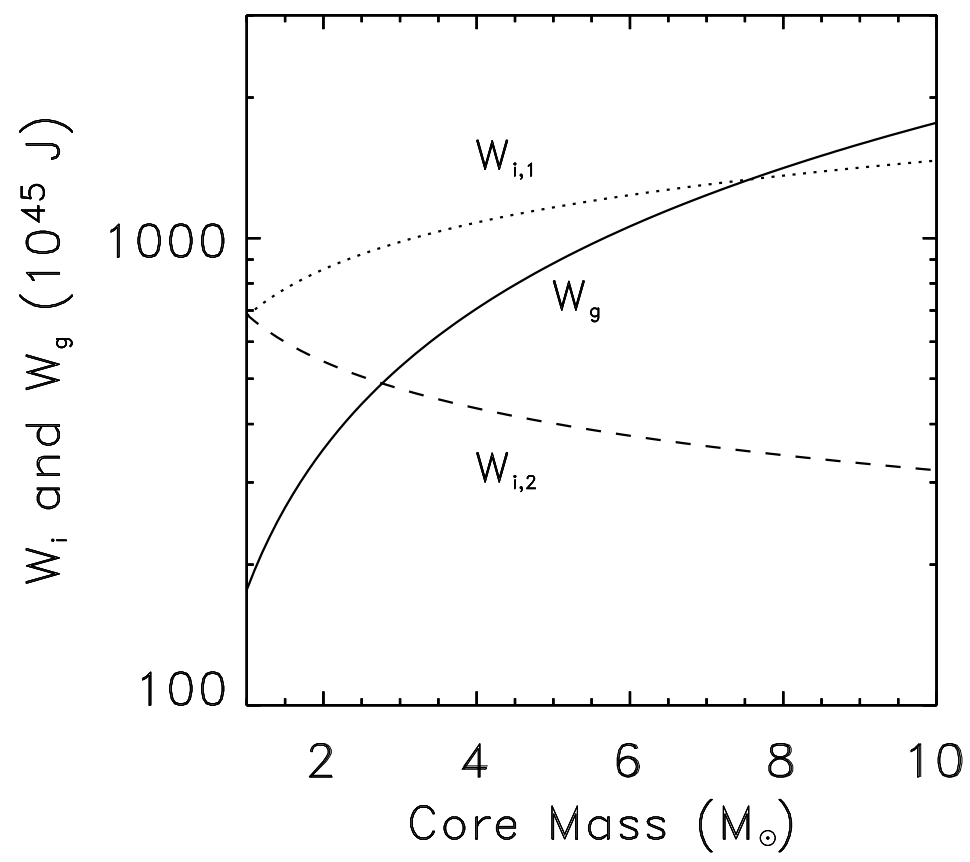

Figure 6. The work done by gravity $\left(W_{g}\right)$ and pressure $P_{i}\left(W_{i, 1}\right.$ for relativistic and $W_{i, 2}$ for non-relativistic) $v s$. the core mass when the core radius collapses to $B$ [47].

Therefore, in general, a star with a collapsing core above the upper mass limit will directly collapse into a black hole, including the mantle material. In this case, no supernova explosion occurred, and the formed black hole includes the entire star with a mass usually tens of solar masses or more. A star with a collapsing core below the lower mass limit will form a neutron star after the mantle, including the core crust, is exploded out, due to the gravitational field shielding or turning off. The formed neutron star can further evolve into a black hole when it accretes enough matter from a companion star, such that the 
total mass exceeds the lower limit. The formed black hole includes the masses of the neutron star and the companion star. A star with a collapsing core below the upper limit, but above the lower limit, will form a neutron star first and, then, as it cools, collapse further into a black hole with a mass below the upper limit. The cooling period of neutron stars can be hundreds of years up to millions of years [69]. The compact object formed from SN1979C should be a neutron star at the moment of its formation [70] and should collapse into a black hole as it cools, if its mass exceeds the lower limit.

In accord with the Schwarzschild solution of the Einsteinian general relativity, the lower and upper mass limits for the formation of a Schwarzschild black hole are much lower than those in the KK theory. This implies that a collapsing star forms a strong gravitational Schwarzschild black hole more easily than a gravitationless KK black hole. This can explain the observations that some very massive stars with masses over 40 solar masses, after supernova explosions, formed neutron stars rather than the expected black holes [48-50].

A black hole that has no gravity at the surface may generate a jet more easily than a black hole that has a strong gravitational field. Accreting material near the surface will be subjected to a strong pressure force along the direction perpendicular to the accreting disc (or parallel to the z-axis) when the gravity is turned off. The strong z-component pressure force can accelerate the material to become relativistic jet flows along the polar magnetic field lines. A magnetohydrodynamic model with the gravitational field shielding effect may simulate the physical process of jet generation.

The gravitationless black hole model does not violate Penrose's cosmic censorship conjecture, because its surface is also an infinite redshift surface $\left(1+Z=\left(-g_{00}^{E}\right)^{-1 / 2}=\phi^{3 / 2} \rightarrow \infty\right.$, as $\left.r \rightarrow B\right)$, or an event horizon [45]. This implies that the singularity is not naked, and even light cannot escape from the gravitationless KK black hole, similar to the Schwarzschild black hole. Particle motion around the KK black hole will be studied and simulated in future according to the motion equation of the 5D fully-covariant KK theory with a scalar field.

\subsection{Space Polarization}

Figure 1d has shown that the electric field of a charged compact object significantly derives from the Coulomb electric field. The reason for this derivation is the polarization of the space by the strong scalar field. To explore this space polarization in more detail, we define the dielectric constant (or the relative permittivity) as,

$$
\epsilon_{r} \equiv \frac{E_{c}}{E}=\phi^{3} \exp \left(\frac{\lambda-\nu}{2}\right)
$$

The relative permittivity $\epsilon_{r}$ depends on the scalar field $\phi$ and the gravitational field metric functions $e^{\lambda}$ and $e^{\nu}$. Therefore, $\epsilon_{r}$ is a function of $\alpha, m$ and $r$. If $\epsilon_{r}>1$ (i.e., $E<E_{c}$ ), we say that the space is polarized.

The polarization coefficient is usually defined by:

$$
\delta_{p} \equiv \frac{E_{c}-E}{E_{c}}=1-\frac{1}{\epsilon_{r}} .
$$

Here, the polarization coefficient is similar to the dielectric susceptibility, which is defined by $\chi \equiv \epsilon_{r}-1=\left(E_{c}-E\right) / E$. The polarization coefficient is in the range of $0 \leq \delta_{p} \leq 1$. The space or vacuum is not polarized when $\delta_{p}=0$ (or $\epsilon_{r}=1$ ) and completely polarized when $\delta_{p}=1$ (or $\epsilon_{r}=\infty$ ). 
Figure 7 plots the relative permittivity $\epsilon_{r}$ as a function of $r / B$ for a charged object with $\alpha=0.1$, 1, 10, 100, 1,000. Figure 8 plots the relative permittivity $\epsilon_{r}$ as a function of radial distance $r$ for a charged compact star with 1.5 solar masses and a charge in the range of $\alpha=0.1-1,000$. It is seen that the electric field asymptotically approaches the Coulomb's electric field (i.e., $\epsilon_{r} \rightarrow 1$ or $\delta_{p} \rightarrow 0$ ) when $r$ is sufficiently large or approaches infinity. When $r$ tends to be small, however, the electric field significantly deviates from the Coulomb's electric field (i.e., $\epsilon_{r} \gg 1$ or $\delta_{p} \rightarrow 1$ ), because the vacuum space is extensively polarized by the strong scalar field. When $r$ tends to $B$, the dielectric coefficient approaches to infinity, and the electric field becomes weak as compared with the strength of the Coulomb's electric field, especially when the object is highly charged. In the limit case of $\epsilon_{r}=\infty$, we say that the vacuum space is completely polarized by the extreme gravitational and scalar fields. It should be noted that a big deviation at $r \sim B$ still exists, even if the object is weakly charged $(\alpha<<1)$. The deviation increases as the charge increases. For instance, at $\alpha=100$ and $r=100 \mathrm{~km}$, the electric field is only $1 \%$ of the Coulomb electric field. The electric field is so weak compared with the strength of the Coulomb electric field, and the vacuum space is almost completely polarized, especially when the object is highly charged. If the compact star is weakly charged, the deviation is negligible when $r>30$ $\mathrm{km}$, but can be increased by about ten times when $r$ decreases to be about $10 \mathrm{~km}$.

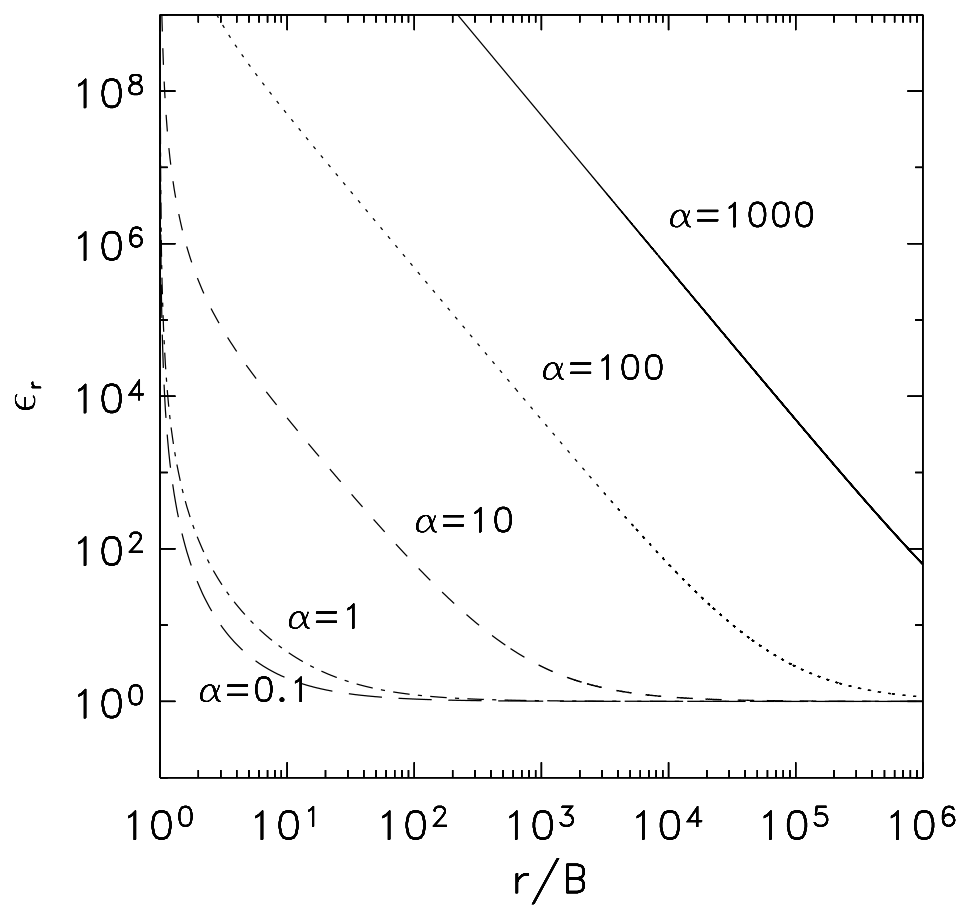

Figure 7. Relative dielectric coefficient $\epsilon_{r}$ or electric field ratio $E_{c} / E$ versus the normalized radial distance $r / B$ for a charged object with $\alpha=0.1,1,10,100,1,000$, respectively.

Using the Coulomb electric field, Ray et al. [58] obtained the maximum amount of charge in a compact star by $Q \sim \sqrt{G} M$, which is $Q \sim 2.5 \times 10^{20} \mathrm{C}$ or $\alpha \sim 0.5$ if $M=3 \times 10^{33} \mathrm{~g}$. In the KK theory with a scalar field, the extensive polarization of the space by the strong scalar field significantly decreases the electric field from the Coulomb electric field around the charged compact star (see Figure $2 d$ of [45] for the scalar field). Therefore, to produce an electric field capable of balancing the radiation dragging between electrons and ions, a compact star requires more electric charges. In other words, the 
maximum amount of charge in a compact star can be significantly higher than that predicted with the Coulomb force if the effect of the scalar field is considered.

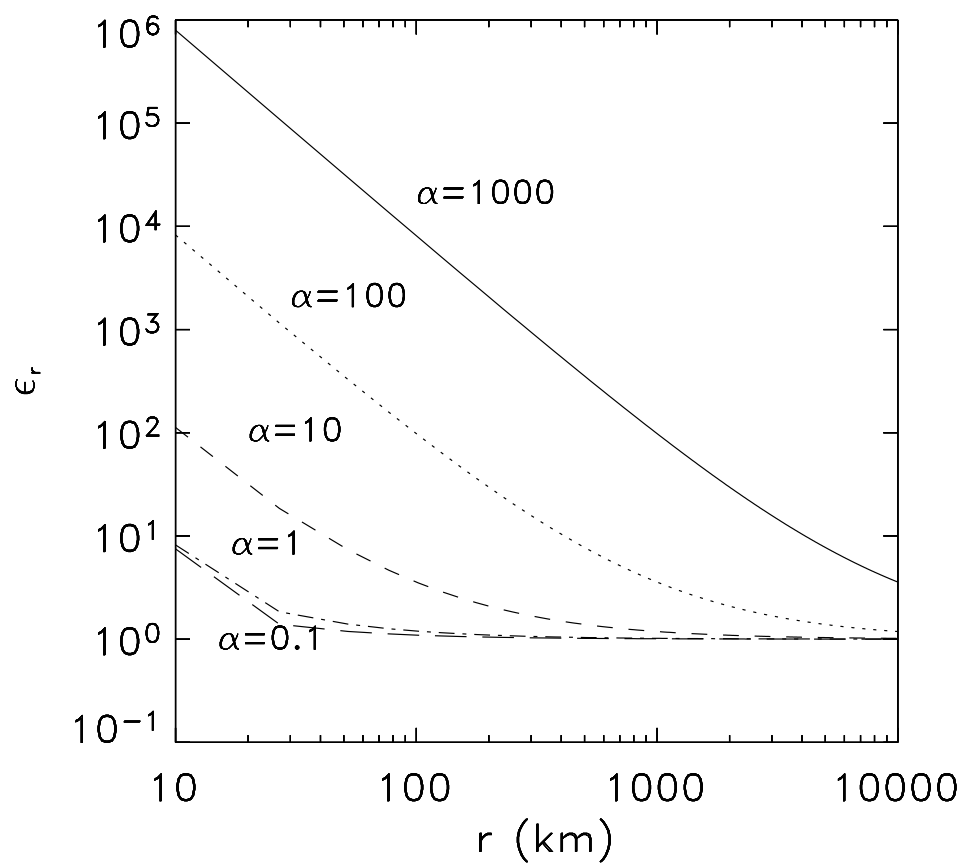

Figure 8. Relative dielectric coefficient $\epsilon_{r}$ or electric field ratio $E_{c} / E$ versus the radial distance $r$ for a charged compact star with 1.5 solar masses and $\alpha=0.1,1$, $10,100,1,000$, respectively.

\section{Discussions and Conclusions}

The 5D fully-covariant $\mathrm{KK} \Phi$ theory can also be applied to develop a cosmology by modifying the Friedmann equation. The scalar field in the 5D gravity plays the role of dark energy for the acceleration of the universe expansion [71,72]. Recently, we have also, in accord with the 5D fully-covariant KK $\Phi$ theory, developed a new mass-radius relation that perfectly explained the new observations of neutron stars, especially those with greater masses and/or smaller radii [73-77]. From this 5D fully-covariant KK $\Phi$ theory, we can have a modified Friedmann equation for cosmology, as well as a modified Tolman-Oppenheimer-Volkoff (TOV) equation for neutron star. These modified equations will reveal the roles of the scalar field in cosmology and astrophysics.

The 5D fully-covariant $\mathrm{KK} \Phi$ theory is a classical unification of gravitational, electromagnetic and scalar fields. In comparison with other 5D KKФ theories and 4D scalar-tensor theories, it has three essential advantages: (1) there does not exist any unknown parameter, such as a scalar charge $s$ or a coupling constant $\omega$, and, thus, it can quantitatively be compared with observations; (2) it is equivalent to the 4D Einstein's general relativity, when the fields are weak and the matter is neutral, and, thus, also passes the fundamental tests, such as the gravitational redshift of light, precession of a planet's perihelion, deflection of light by the Sun, delay of radar echoes, and so on; and (3) it predicts many new effects, when the fields are strong, such as the space polarization, electric redshift, gravitational fielding (or space flattening) etc., and, thus, is able to remodel cosmology and strong field astrophysics. To unify more fundamental interactions, such as weak and strong forces, we must extend the dimension of the 
spacetime from 5D to $(n+4) \mathrm{D}$ to include non-Abelian Yang-Mills fields. To develop a quantum theory of unification, we shall study quantum wave equations in higher dimensions [78].

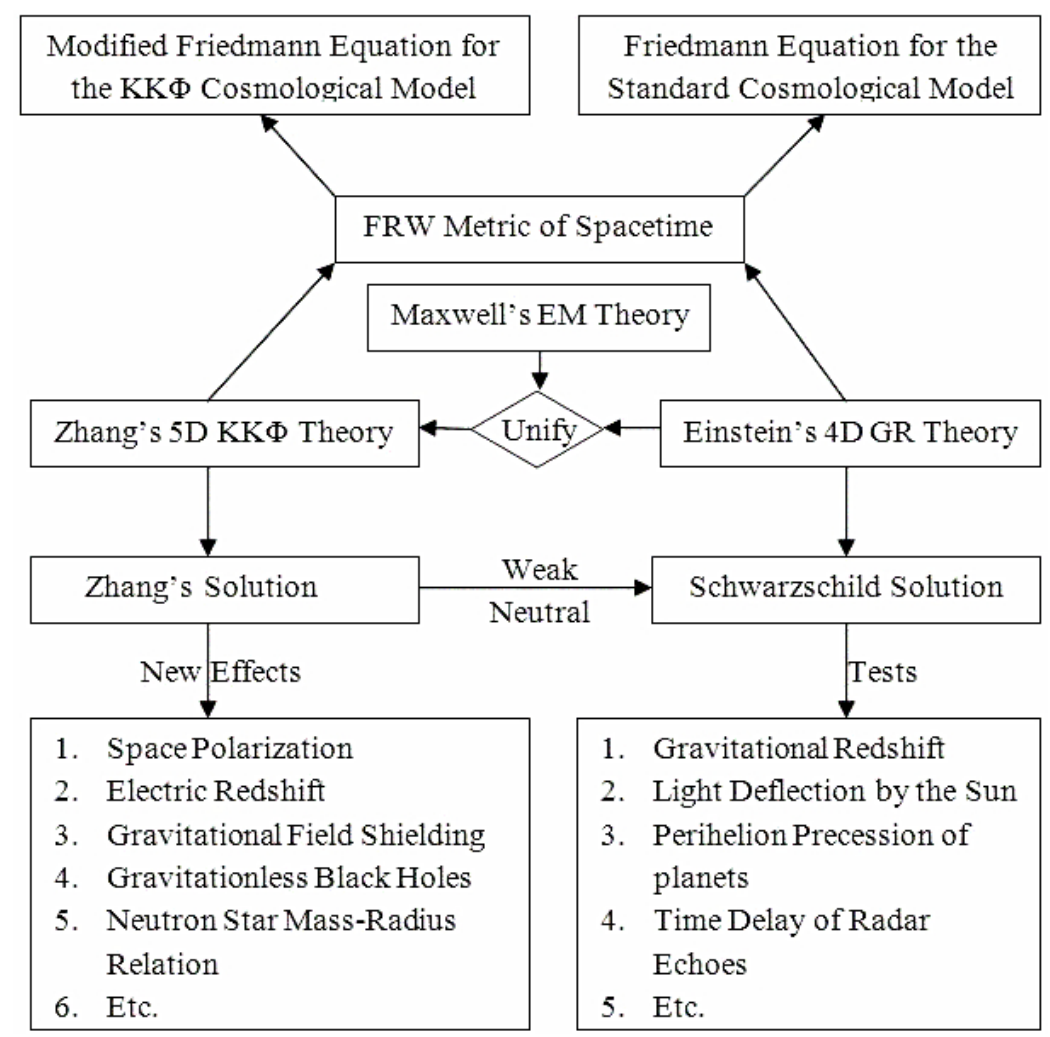

Figure 9. Various aspects of the 5D fully-covariant $K K \Phi$ theory with a scalar that unified the 4D Einstein's general relativity and Maxwell's electromagnetic theory. Zhang's field solution reduces to the Schwarzschild solution in the Einstein frame when the fields are weak and matter is neutral and, thus, experimentally well tested with the fundamental measurements. In the strong field case, especially when matter is significantly charged, the field solution predicts results with new effects, such as the space polarization, electric redshift, gravitational field shielding, and so on. Furthermore, a 5D fully covariant $\mathrm{KK} \Phi$ cosmology with a scalar field dark energy can be developed by using the Friedmann-Lemaitre-Robertson-Walker metric of spacetime.

As a consequence, we have comprehensively reviewed the 5D fully-covariant $\mathrm{KK} \Phi$ theory and its application to high energy and strong field astrophysics. This model uniquely describes the action of matter also in 5D spacetime, which allows us to analytically derive the fifteenth component of the 5D energy-momentum tensor of matter without assuming as usually an unknown parameter, called the scalar charge, and thus, this guarantees that the developed 5D fully-covariant $\mathrm{KK} \Phi$ theory does not include any unknown parameter and undetermined constant. The gravitational, electromagnetic and scalar field equations that are obtained from splitting the 5D Einsteinian field equation of the 5D fully-covariant KK $\Phi$ theory were solved with an exact static spherically-symmetric external field solution and a weak internal field solution. All integration constants in the exact external field solution were completely determined with a perfect set of simple numbers and parameters that only depend on the mass and electric charge. In the Einstein frame, the exact field solution obtained from the 5D fully-covariant KK $\Phi$ 
theory reduces to the Schwarzschild solution of Einstein's general relativity when the matter is neutral and the fields are weak. This guarantees that the fundamental tests of Einstein's general relativity in the cases of the weak field are also the tests of the 5D fully-covariant $\mathrm{KK} \Phi$ theory. In the strong field, especially when the matter is charged, however, the results from the $5 \mathrm{D}$ fully-covariant $\mathrm{KK} \Phi$ theory are significantly different from Einstein's general relativity. Recently, the author has developed a new redshift mechanism, called electric redshift, a new supernova explosion mechanism, called gravitational field shielding, and a gravitationless black hole model, in accord with the exact solution of this 5D fully-covariant Kaluza-Klein theory with a scalar field. Figure 9 summarizes the various aspects of the 5D fully-covariant KK theory with a scalar field. This paper has provided an overall review of this 5D fully-covariant theory of gravitation, including the development of the theory, the properties of the solution and applications in astrophysics.

\section{Acknowledgments}

This work was supported by NASA Experimental Program to Stimulate Competitive Research (EPSCoR) (NNX07AL52A), Alabama A\&M University Title III programs and the National Natural Science Foundation of China (G40890161). The author thanks Zi-Tian Yang, Shou-Li Peng and Lin Chen from Yunnan University; Yukiharu Ohsawa and Yoshinosuke Telashima from Nagoya University; and Xiao-Qing Li from the Purple Mountain Observatory. He is also grateful to the reviewers and editors for their scientific reviews and editions, and to Susan Phelan for her English assistance.

\section{Conflicts of Interest}

The authors declare no conflict of interest.

\section{References}

1. Kaluza, T. On the problem of unity in physics. Sitz. Preuss. Aklad. Wiss. (Phys. Math.) 1921, K1, 966-972.

2. Klein, O. Quantum theory and five-dimensional theory of relativity. Z. Phys. 1926, 37, 895-906.

3. Klein, O. The atomicity of electricity as a quantum theory law. Nature 1926, 118, 516-516.

4. Einstein, A.; Bergmann, P.G. On a generalization of Kaluza's theory of electricity. Ann. Math. 1938, 34, 683-6701.

5. Bergmann, P.G. Introduction to the Theory of Relativity; Prentice-Hall, Inc.: New York, NY, USA, 1942.

6. Einstein, A.; Pauli, W. On the Non-existence of regular stationary solutions of relativistic field equations. Ann. Math. 1942, 44, 131-137.

7. Souriau, J.M. Five-dimensional relativity. Nuovo Cimento 1963. 30, 565-578.

8. Dirac, P.A.M. The cosmological constants. Nature 1937, 139, 323-323.

9. Jordan, P. Erweiterung der projektiven relativitastheorie. Ann. Phys. 1947, 436, 219-228.

10. Thiry, Y.R. Les equations de la theorie unitaire de Kaluza. C. R. Hebd. Seanc. Acad. Sci. 1948, 226, 216-218. (In French)

11. Bergmann, P.G. Unified field theory with fifteen field variables. Ann. Math. 1948, 49, 255-264. 
12. Nodvik, J.D. Suppression of singularities by the g55 field with mass and classical vacuum polarization in a classical Kaluza-Klein theory. Phys. Rev. Lett. 1985, 55, 2519-2522.

13. Dragilev, V.M. Vacuum polarization of a scalar field in anisotropic multidimensional cosmology. Theor. Math. Phys. 1990, 84, 887-893.

14. Pavsic, M. Solution of the Planck mass problem in Kaluza-Klein theories. J. Phys. G Nucl. Part. Phys. 1982, 8, L89-L92.

15. Leibowitz, E.; Rosen, N. Five-dimensional relativity theory. Gen. Relat. Gravit. 1973, 4, 449-474.

16. Vladimirov, Y.S.; Kislov, V.V. Variation of $\mathrm{e} / \mathrm{m}$ in the five-dimensional theory of gravitation, electromagnetism, and scalar field. Vestn. Mosk. Univ. Fiz. 1982, 23, 18-21.

17. Kovacs, D. The geodesic equation in five-dimensional relativity theory of Kaluza-Klein. Gen. Relat. Gravit. 1984, 16, 645-655.

18. De Leon, J.P. Mass and charge in brane world and noncompact Kaluza-Klein theories in five-dimensions. Gen. Relat. Gravit. 2003, 35, 1365-1384.

19. Gegenberg, J.; Kunstatter, G. The motion of charged particles in Kaluza-Klein space-time. Phys. Lett. A 1984, 106, 410-414.

20. Hsu, R.R.; Yeung, W.B. Solution for the five-dimensional general relativity that simulates a charged macroscopic object in four dimensions. Lett. Nuovo Cimento 1984, 42, 352-356.

21. Moose, A.E.; Ahner, H.F. Inequivalence of five-dimensional relativity and Brans-Dicke theory. Phys. Rev. D 1982, 26, 1275-1280.

22. Sorkin, R.D. Kaluza-Klein monopole. Phys. Rev. Lett. 1983, 51, 87-90.

23. Gross, D.J.; Perry, M.J. Magnetic monopoles in Kaluza-Klein theories. Nucl. Phys. B 1983, 226, 29-48.

24. Perry, M.J. Non-abelian Kaluza-Klein monopoles. Phys. Lett. B 1984, 137, 171-174.

25. Lou, S. L. Magnetic monopole in five-dimensional Kaluza-Klein theory. Lett. Nuovo Cimento 1985, 42, 192-194.

26. Maeda, K.I. Effect of particle creation on Kaluza-Klein cosmologies. Phys. Rev. D 1984, 30, 2482-2494.

27. Randjbar-Daemi, S.; Salam, A.; Strathdee, J. On Kaluza-Klein cosmology. Phys. Lett. B 1984, 135, 388-392.

28. Bailin, D.; Love, A. Kaluza-Klein theories in twelve dimensions. Nucl. Phys. B 1985, 254, 543-554.

29. Barrow, J.D.; Stein-Schabes, J. The stability of some Kaluza-Klein cosmological models. Phys. Lett. B 1986, 167, 173-177.

30. Okada, Y. Evolution of the Kaluza-Klein universe. Nucl. Phys. B 1986, 264, 197-220.

31. Grøn, Ø. Classical Kaluza-Klein description of the hydrogen atom. Nuovo Cimento B 1986, 91, 57-66.

32. Rindani, S.D.; Sivakumar, M. Consistent theory of massive spin-3/2 particle with electromagnetic and gravitational interaction by Kaluza-Klein reduction. J. Phys. G Nucl. Part. Phys. 1986, 12, 1335-1340.

33. Chodos, A.; Detweiler, S. spherically-symmetric solutions in five-dimensional general relativity. Gen. Relat. Gravit. 1982, 14, 879-890. 
34. Davison, A.; Owen, D. A. Black holes as windows to extra dimensions. Phys. Lett. B 1985, 155, 247-250.

35. Angus, I.G. spherically-symmetric solutions in Abelian Kaluza-Klein theories. Nucl. Phys. B 1986, 264, 349-363.

36. De Leon, J.P.; Wesson, P.S. Exact solutions and the effective equation of state in Kaluza-Klein theory. J. Math. Phys. 1993, 34, 4080-4090.

37. Liu, H.; Wesson,P.S. A class of Kaluza-Klein soliton solutions. Phys. Lett. B 1996, 381, 420-422.

38. Liu, H.; Wesson, P.S. The physical properties of charged five-dimensional black holes. Class. Quantum Gravity 1997, 14, 1651-1663.

39. Abbott, R.B.; Barr, S.M.; Ellis, S.D. Kaluza-Klein cosmologies and inflation. Phys. Rev. D 1985, 30, 720-727.

40. Wesson, P.S. A physical interpretation of Kaluza-Klein cosmology. Astrophys. J. 1992, 394, 19-24.

41. Zhang, T.X. Static spherically-symmetric solution of Kaluza-Klein theory with five-dimensionally complete covariance and its experimental tests. Master's Thesis, Yunnan University, Kunming, China, 1987.

42. Yang, Z.T. Kaluza-Klein theory. In Lecture Notes; Yunnan University: Kunming, China, 1986.

43. Deng, S.H. Conservation laws and quantities in five-dimensional Kaluza-Klein theory. Master's Thesis, Yunnan University, Kunming, China, 1987.

44. Zhang, T.X. Kaluza-Klein theory with five-dimensionally complete covariance. J. Nanchang Univ. Nat. Sci. 1993, 17, 63-70.

45. Zhang, T.X. Electric Redshift and Quasars. Astrophys. J. Lett. 2006, 636, L61-L64.

46. Zhang, T.X. Gravitational field shielding and supernova explosion. Astrophys. J. Lett. 2010, 725, L117-L120.

47. Zhang, T.X. Gravitationless black holde. Astrophys. Space Sci. 2011, 330, 157-163.

48. Figer, D.F.; Mackenty, J.W.; Robberto, M.; Smith, K.; Najarro, F.; Kudritzki, R.P.; Herrero, A. Discovery of an extraordinarily massive cluster of red supergiants. Astrophys. J. 2006, 643, 1166-1179.

49. Muno, M.P.; Clark, J.S.; Crowther, P.A.; Dougherty, S.M.; de Grijs, R.; Law, C.; McMillan, S.L.W.; Morris, M.R.; Negueruela, I.; Pooley, D.; et al. A neutron star with a massive progenitor in Westerlund 1. Astrophys. J. Lett. 2006, 636, L41-L44.

50. Ritchie, B.W.; Clark, J.S.; Negueruela, I.; Langer, N. A VLT/FLAMES Survey for Massive Binaries in Westerlund 1. II. Dynamical Constraints on Magnetar Progenitor Masses from the Eclipsing Binary W13. Astron. Astronphys. 2010, 520, A48.

51. Overduin, J.M.; Wesson, P.S. Kaluza-Klein gravity. Phys. Rep. 1997, 283, 303-378.

52. Brans, C.; Dicke, R.H. Mach's Principle and a Relativistic Theory of Gravitation. Phys. Rev. 1961, 124, 925-935.

53. Weinberg, S. Gravitation and Cosmology; Wiley: New York, NY, USA, 1980.

54. Schwarzschild, K. Ober das gravitationsfeld eines massenpunktes nach der Einsteinschen theorie. Sitz. Preuss. Akad. Wiss. 1916, 1, 189-196.

55. Macias, A.; Garcia, A. Jordan Frame or Einstein Frame? Gen. Relat. Gravit. 2001, 33, 889-899. 
56. Flanagan, E.F. The conformal frame freedom in theories of gravitation. Class. Quantum Gravity 2004, 21, 3817-3829.

57. Whinnett, A.W.; Torres, D.F. Charged scalar-tensor boson stars: Equilibrium, stability, and evolution. Phys. Rev. D 1999, 60, 104050.

58. Ray, S.; Espíndola, A. L.; Malheriro, M.; Lemos, J.P.; Zanchin, V.T. Electrically charged compact stars and formation of charged black holes. Phys. Rev. D 2003, 68, 084004.

59. Kutschera, M.; Wójcik, W. Proton impurity in the neutron matter: A nuclear polaron problem. Phys. Rev. C Nucl. Phys. 1993, 47, 1077-1085.

60. Baiko, D. A.; Haensel, P. Cooling neutron stars with localized protons. Astron. Astrophys. 2000, 356, 171-174.

61. López-Corredoira, M.; Gutiérrez, C.M. The field surrounding NGC 7603: Cosmological or non-cosmological redshifts? Astron. Astrophys. 2004, 421, 407-423.

62. Arp, H. Quasars, Redshifts, and Controversies; Cambridge University Press: Cambridge, UK, 1988.

63. Schild, R.E.; Leiter, D.J.; Robertson, S.L. Observations Supporting the Existence of an Intrinsic Magnetic Moment inside the Central Compact Object within the Quasar Q0957+561. Astron. J. 2006, 132, 420-432.

64. Jadhav, M.; Zhang, T.X.; Winebarger, A. Electric Redshift in Jordan and Einstein Frames. Bull. Am. Astron. Soc. 2010, 42, 441-441.

65. Zhang, B.J.; Zhang, T.X.; Guggilla, P.; Dokhanian, M. Gravitational field shielding by scalar field and type II superconductors. Prog. Phys. 2013, 1, 69-73.

66. Yakovlev, D.G.; Gnedin, O.Y.; Kaminker, A.D.; Levenfish, K.P.; Potekhin, A.Y. Neutron star cooling: Theoretical aspects and observational constraints. Adv. Space Res. 2004, 33, 523-530.

67. Cameron, A.G. Neutron star models. Astrophys. J. 1959, 130, 884-894.

68. Fechner W.B.; Joss, P.C. Quark stars with realistic equation of state. Nature 1978, 274, 347-349.

69. Slane, P.O.; Helfand, D. J.; Murphy, S.S. New Constraints on Neutron Star Cooling from Chandra Observations of 3C 58. Astrophys. J. Lett. 2002, 571, L45-L49.

70. Pacini, F.; Salvati, M. Radio emission from very young supernova remnants-The case of SN 1979c. Astrophys. J. Lett. 1981, 245, L107-L108.

71. Jadhav, M.; Zhang, T.X.; Winebarger, A. Modified Friedmann equation with a scalar field. In Proceedings of the Alabama Academy of Science (AAS) 87th Annual Meeting, Huntsville, Alabama, USA, 30 March - 1 April, 2010.

72. Jadhav, M.; Zhang, T.X.; Winebarger, A. Modified Friedmann equation with a scalar field. In AAMU STEM Day; Alabama A\&M University: Normal, AL, USA, 2010; Abstract 94, 66-66. Available online: http://www.docstoc.com/docs/122056761/ STEM-DAY-STEM-DAY-Physics-Alabama (accessed on 10 December 2014)

73. Guver, T.; Ozel, F.; Cabrera-Lavers, A.; Wroblewski, P. The Distance, Mass, and Radius of the Neutron Star in 4U 1608-52. Astrophys. J. 2010, 712, 964-973.

74. Guver, T.; Wroblewski, P.; Camarota, L.; Ozel, F. The Mass and Radius of the Neutron Star in 4U 1820-30. Astrophys. J. 2010, 719, 1807-1812. 
75. Guillot, S.; Rutledge, R.E.; Brown, E.F. Neutron star radius measurement with the quiescent low-mass X-ray binary U24 in NGC 6397. Astrophys. J. 2011, 732, 88, doi:10.1088/0004-637X/732/2/88.

76. Demorest, P.B.; Pennucci, T.; Ransom, S.M.; Roberts, M.S.E.; Hessels, J.W.T. A two-solar-mass neutron star measured using Shapiro delay. Nature 2010, 467, 1081-1083.

77. Zhang, B.J.; Zhang, T.X.; Gugilla, P.; Dokhanian, M. Neutron star mass-radius relation with gravitational field shielding by a scalar field. Res. Astron. Astrophys. 2013, 13, 571-578.

78. Dong, S.H. Wave Equations in Higher Dimensions; Springer: New York, NY, USA, 2011.

(c) 2014 by the author; licensee MDPI, Basel, Switzerland. This article is an open access article distributed under the terms and conditions of the Creative Commons Attribution license (http://creativecommons.org/licenses/by/4.0/). 